\title{
6A3-5/Osa2 is an Early Activated Gene Implicated in the Control of Vascular Smooth Muscle Cell Functions
}

\author{
Gwenaele Garin,, ${ }^{1}$ Kazem Zibara, ${ }^{1,2}$ Frederick Aguilar, ${ }^{3}$ Ming Lo, ${ }^{3}$ Adam Hurlstone, ${ }^{4}$ Robin Poston, ${ }^{5}$ and \\ John L. Mcgregor1, 2,5 \\ ${ }^{1}$ INSERM XR331, Faculté of Médicine Laënnec, Lyon 69372, France \\ ${ }^{2}$ Genomics and Atherothrombosis, Thrombosis Research Institute, London SW3 6LR, UK \\ ${ }^{3}$ Département de Physiologie et Pharmacologie Clinique, Faculté de Pharmacie, Université Lyon 1, Lyon, France \\ ${ }^{4}$ Hubrecht Laboratory, Netherlands Institute for Developmental Biology, Utrecht, The Netherlands \\ ${ }^{5}$ Center for Cardiovascular Biology and Medicine, King's College, University of London, UK
}

Received 17 April 2006; Revised 11 July 2006; Accepted 17 July 2006

Vascular smooth muscle cells (VSMC) growth plays a key role in the pathophysiology of vascular diseases. However, the molecular mechanisms controlling gene transcription in VSMC remain poorly understood. We previously identified, by differential display, a new gene (6A3-5) overexpressed in proliferating rat VSMC. In this study, we have cloned the full-length cDNA by screening a rat foetal brain cDNA library and investigated its functions. The 6A3-5 protein shows 4 putative conserved functional motifs: a DNA binding domain called ARID (AT-rich interaction domain), two recently described motifs (Osa Homology Domain), and a nuclear localization signal. The deduced protein sequence was observed to be $85 \%$ identical to the recently described human Osa2 gene. Immunolabelling, using an anti-6A3-5/Osa2 monoclonal antibody, showed a nuclear localization of the 6A3-5/Osa2 protein. In addition, PDGF upregulated 6A3-5/Osa2 expression at both the transcript and protein levels in a dose and time-dependent fashion. The pattern of upregulation by PDGF was reminiscent of the early responsive gene c-fos. The PDGF-induced upregulation of 6A3-5/Osa2 and proliferation of VSMC were significantly inhibited in a dose and sequence-dependent fashion by an antisense, but not by sense, scrambled or mismatched oligonucleotides directed against 6A3-5/Osa2. In VSMC of aortas derived from hypertensive (LH) rats, 6A3-5/Osa2 is overexpressed as compared to that in normotensive (LL) rats. The 6A3-5/Osa2-gene expression is downregulated by an ACE inhibitor and upregulated by exogenous AngiotensinII in LH rats. In summary, these results indicate that $6 \mathrm{~A} 3-5 / \mathrm{Osa} 2$ is an early activated gene that belongs to a new family of proteins involved in the control of VSMC growth.

Copyright (c) 2006 Gwenaele Garin et al. This is an open access article distributed under the Creative Commons Attribution License, which permits unrestricted use, distribution, and reproduction in any medium, provided the original work is properly cited.

\section{INTRODUCTION}

Vascular smooth muscle cell (VSMC) growth plays a critical role in different pathological conditions such as atherosclerosis [1] and its clinical complications. Indeed, development of these vascular diseases is associated with a loss of vascular contractility counterbalanced by an increase of VSMC migration, proliferation, matrix secretion, and, in some cases, hypertrophy [2]. Different agonists modulate VSMC phenotype and activities in the vessel wall. For example, platelet-derived-growth factor (PDGF), particularly PDGF-BB, stimulates both proliferation and migration [3]. AngiotensinII (AngII), the active biological peptide of the renin-angiotensin system, has potent vasoconstrictor actions

The first two authors are considered joint first authors of this work. and is directly involved in the development of hypertension. AngII induces a multitude of signalling pathways which, depending on the VSMC phenotype, can lead to contraction, hyperplasia, or hypertrophy $[4,5]$. Many transcription factors (such as c-fos [6], Ets-1 [7], $\mathrm{NF} \kappa \mathrm{B}$ [8]) and the subsequent expression of a large number of genes (eg, alphaactin, Collagen IV, MCP-1, Endothelin-1, PDGF-A, TSP-1, bFGF, and PDGF A-chain [9]) are stimulated by AngII. However, the molecular mechanisms controlling gene transcription during these processes remain at this stage poorly understood.

A new gene (6A3-5/Osa2), which is overexpressed in proliferating, rat aortic VSMC, was initially identified by differential display [10]. This partially cloned gene of $1.2 \mathrm{~kb}$, not referenced in Genbank, shares sequences homologies with the ARID (AT-rich interaction domain) transcription modulator family. ARID-containing proteins are involved in the 
control of transcription during cell growth and embryonic development $[11,12]$. However, their precise functions are not fully understood. In the current study, we have cloned the full-length rat $6 \mathrm{~A} 3-5 / \mathrm{Osa} 2 \mathrm{cDNA}$ and characterized its deduced protein sequence as a member of the ARID family. Moreover, the knock-down of 6A3-5/Osa2 expression, which is overexpressed in PDGF-dose and time-dependant manner, resulted in a significant reduction of VSMC proliferation. In vivo work showed that $6 \mathrm{~A} 3-5 / \mathrm{Osa} 2$ is overexpressed in SMC of aortas derived from hypertensive (Lyon hypertensive, LH) but not normotensive (Lyon low-blood pressure, $\mathrm{LL})$ rats. The 6A3-5/Osa2-gene expression is downregulated by an ACE inhibitor and upregulated by exogenous AngII in hypertensive rats.

\section{MATERIALS AND METHODS}

\section{Isolation of a full-length 6A3-5 rat CDNA}

A 6A3-5 full-length cDNA was cloned by screening a rat foetal brain cDNA library (Origene Technologies, Inc) using primers generated from a previously derived partial sequence (Genbank accession number: AJ005202) [10], combined with in silico analysis of genome databases. BlastA (NCBI) and multiple alignments performed using ClustalW (EBI) were used for assessing sequence homologies.

\section{Cell culture}

Primary human and rat VSMC were cultured as previously described [13]. VSMC, at $80 \%$ of confluence, were serum starved for $48 \mathrm{~h}$ and stimulated by PDGF-BB. Dose-effect $(0$ to $20 \mathrm{ng} / \mathrm{ml})$ and time-response $(0,2,4,8$, or $24 \mathrm{~h})$ experiments were performed on human VSMC. Following treatment, VSMC are harvested in Trizol or in lysis buffer $(1 \%$ of $10 \mathrm{mM}$ aprotinin, $10 \mathrm{mM}$ leupeptine, $10 \mathrm{mM}$ EDTA, and $1 \mathrm{mM}$ phenylmethylsulfonyl fluoride, $25 \mathrm{mM}$ Tris $\mathrm{pH} 7.6$, $150 \mathrm{mM} \mathrm{NaCl}$, and 1\% Triton X100).

\section{Immunofluorescence}

After fixation and permeabilization (100\% methanol at $-20^{\circ} \mathrm{C}$ during $\left.5 \mathrm{~min}\right)$, nonspecific sites were blocked (PBS/3\%BSA) for 1 hour at $25^{\circ} \mathrm{C}$. The primary antibody (6H3 anti-Osa2 hybridoma supernatant (1: 5) [14], mouse anti- $\alpha$-actin monoclonal antibody $(1: 100)$ or a rabbit antiNFKB polyclonal antibody (1 : 100), Dako) was incubated for 2 hours at $25^{\circ} \mathrm{C}$. After 3 washing steps, VSMC were incubated in the blocking solution with an appropriate secondary antibody-FITC-conjugated (Dako) for 1 hour at $37^{\circ} \mathrm{C}$. After 4 washing steps, coverslips were mounted and analysed by fluorescence microscopy.

\section{Northern blot}

Total RNA was isolated according to the Trizol procedure. Northerns were performed as previously described [15]. The abundance of 6A3-5/Osa2 mRNA was normalized with respect to $18 \mathrm{~S}$ rRNA and the ratio expressed in arbitrary units (au).

\section{Western blot}

Nitrocellulose membrane bearing electrotransfered proteins $(30 \mu \mathrm{g})$, separated on $7 \%$ SDS-polyacrylamide gels, were blocked for 4 hours at $37^{\circ} \mathrm{C}$ with TBS/ $0.05 \%$ Tween $20 / 3 \%$ gelatine, and incubated overnight at $4^{\circ} \mathrm{C}$ with an anti-Osa2 antibody $(6 \mathrm{H} 3,1: 5[14])$. A swine anti-mouse antibody, conjugated to horseradish peroxidase (Bio-Rad), was then used with a chemiluminescent technique (ECL kit ${ }^{\mathrm{TM}}$, Amersham). Expression level of 6A3-5/Osa2 protein was estimated by Quantity One tool (Bio-Rad) and normalized with Coomassie blue staining.

\section{Gene knock-down by antisense oligonucleotides}

The sequences and locations of the generated oligonucleotides targeted against human Osa2 cDNA AF468300 are summarized in Table 1. For transfection experiments, VSMC at $60-70 \%$ of confluence were serum-starved for $48 \mathrm{~h}$ and then incubated with 25-200 nM ODN at concentration in serum- and antibiotic-free MEM medium in the presence of oligofectamine (Invitrogen). After 4 hours, VSMC were stimulated by PDGF $(20 \mathrm{ng} / \mathrm{ml}$ ) for different periods of time $(0,2$, 4,6 , and $24 \mathrm{~h}$ ) and then harvested in a cell lysis buffer or Trizol. Alternatively, after transfection, VSMC were stimulated by PDGF for 24 hours and used for Bromodeoxyuridine incorporation test (Roche) to estimate cell proliferation.

\section{Animal studies}

Protocols for animals' (Lyon hypertensive (LH) and Lyon low-blood pressure (LL) strains) housing and treatment have been previously detailed [16]. Three groups were used: the first group (controls, $n=8$ ) was untreated and used as controls. The second group (Ace I, $n=8$ ) was treated with an ACE inhibitor, perindopril ( $3 \mathrm{mg} / \mathrm{kg} / \mathrm{d})$, for 4 weeks. The third group (Ace I+ANGII, $n=8$ ) was treated with an ACE inhibitor, perindopril (3 mg/kg/d) and perfused subcutaneously with AngII (200 ng/kg/min) for 4 weeks.

\section{Quantification of 6A3-5/Osa2 mRNA by quantitative-PCR}

Frozen rat aortas were homogenized at $0^{\circ} \mathrm{C}$ in $500 \mu \mathrm{l}$ Trizol and total RNA isolated. Reverse transcription product ( $\mathrm{Su}-$ perscript II, Invitrogen) was used for quantitative real-time PCR (Q-PCR) on an ABIPrism 7900. Q-PCR assay was carried out using the Assay-on-Demand for 6A3-5, calponin, SM22-alpha, and $18 \mathrm{~S}$ mRNA levels were using the comparative $\mathrm{Ct}$ method.

\section{Immunohistochemistry}

Immunohistochemistry was performed on frozen aorta sections $(5 \mu \mathrm{m})$ fixed in acetone as previously described [15] with an anti-Osa2 hybridoma supernatant (6H3) [14] or an anti- $\alpha$-actin (Dako) monoclonal antibody. Primary antibody binding was detected using a secondary antibody 
TABLE 1: Homologies of rat 6A3-5 sequences with ARID proteins. Homologues are divided into two subgroups. The first subgroup, which would define the subfamily called Osa, has members bearing an ARID motif and two OHD domains. The second subgroup indicates different ARID proteins.

\begin{tabular}{|c|c|c|c|c|c|}
\hline & Species & Yeast & Drosophila & Mouse & Human \\
\hline \multirow{12}{*}{ 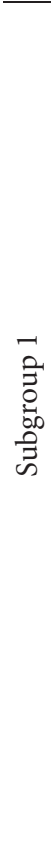 } & Names & Swil & Osa, eyelid & Osal & Osa1, P270, B120, BAF250 \\
\hline & Accesion no & M84390 & AF053091 & AF268912 & AF521670 \\
\hline & Chromosome no & - & 3 & 4 & 1 p35-p36 \\
\hline & cDNA Length & $3027 \mathrm{bp}$ & $10601 \mathrm{bp}$ & $7041 \mathrm{bp}$ & $6418 \mathrm{bp}$ \\
\hline & Protein Length & 825 aa & 2715 aa & 1902 aа & 1999 aа \\
\hline & Functions & $\begin{array}{l}\text { - Member of } \\
\text { yeast SWI/SNF }\end{array}$ & $\begin{array}{l}\text { - Member of Brahma complex } \\
\text { - Antagonize wingless pathways }\end{array}$ & $\begin{array}{l}\text { - Interaction with Brahma } \\
\text { chromatin remodelling } \\
\text { complex }\end{array}$ & $\begin{array}{l}\text { - Member of human swi/snf } \\
\text { - Co-factor of transcriptional } \\
\text { activation by the steroid } \\
\text { hormone receptors }\end{array}$ \\
\hline & Name & nd & nd & nd & Osa2, held/Osa1, KIAA1235 \\
\hline & Accesion no & - & - & - & AF521671 and AF468300 \\
\hline & Chromosome no & - & - & - & $6 q 25.1-q 25.3$ \\
\hline & cDNA Length & - & - & - & $5482 \mathrm{pb}$ \\
\hline & Protein Length & - & - & - & 1740 aa \\
\hline & Functions & - & - & - & $\begin{array}{l}\text { - Member of human swi/snf } \\
\text { - Promotes transcriptional } \\
\text { activation by the steroid } \\
\text { hormone receptors }\end{array}$ \\
\hline \multirow{18}{*}{ 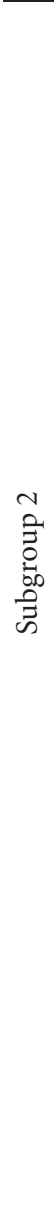 } & Name & nd & Dead Ringer (Dri) & Bright & DRIL-1 \\
\hline & Accesion no & - & U62542 & U60335 & U88047 \\
\hline & Chromosome no & - & - & 10 & $19 \mathrm{p} 13.3$ \\
\hline & cDNA Length & - & $3696 \mathrm{bp}$ & 4842 bp & $2725 \mathrm{pb}$ \\
\hline & Protein Length & - & 901 aa & 601 aa & 593 aa \\
\hline & Functions & - & $\begin{array}{l}\text { - Embryo patterning } \\
\text { - Target sequence: } \\
\text { AGATT/ATAA }\end{array}$ & $\begin{array}{l}\text { - B-Cell activator } \\
\text { - Target sequence: } \\
\text { AGATTAA }\end{array}$ & $\begin{array}{l}\text { - Binds the } \mathrm{pRb} \\
\text { controlled transcription } \\
\text { - Target sequence: } \\
\text { A/GATT/ATAA }\end{array}$ \\
\hline & Name & nd & nd & Mrf2 & Mrf2 \\
\hline & Accesion no & - & - & AF280065 & M733837 (partial sequence) \\
\hline & Chromosome no & - & - & 10 & 10 \\
\hline & cDNA Length & - & - & $3647 \mathrm{bp}$ & - \\
\hline & Protein Length & - & - & 1188 aa & - \\
\hline & Functions & - & - & $\begin{array}{l}\text { - Accumulation of } \\
\text { lipids in postnatal life } \\
\text { - Target sequence: } \\
\text { AATA }(\mathrm{C} / \mathrm{T})\end{array}$ & - \\
\hline & Name & nd & nd & Jumonji & Jumonji \\
\hline & Accesion no & - & - & BC05244 & U57592 \\
\hline & Chromosome no & - & - & - & $6 q 24 . p 23$ \\
\hline & cDNA Length & - & - & 4939 bp & - \\
\hline & Protein Length & - & - & 1324 aa & 1266 aa \\
\hline & Functions & - & - & - Neural embryogenesis & $\begin{array}{l}\text { Highly expressed by neuron } \\
\text { cells during development }\end{array}$ \\
\hline
\end{tabular}


TABLE 2: List of antisens oligonucleotides directed against 6A3-5. Sequence and position (on human homologous held/Osa2) of different ODN directed against 6A3-5. Only ODNAS3 showed significant effects on 6A3-5 expression. Scrambled ODN3 (ODN Scr3) and mismatched (ODN Mis3) as used to test sequence specificity of ODNAS3.

\begin{tabular}{l|cc}
\hline Name & Sequence $5^{\prime}-3^{\prime}$ & Position/AF468300 \\
\hline ODNAs1 & agcttgtcgaacttactggct & $3870-3890$ \\
ODNAs2 & cagcttgtcgaacttactggctt & $3869-3889$ \\
ODNAs3 & tgggatctgcccatg & $57-71$ \\
ODN Scr3 & agctcggttcacggt & - \\
ODN Mis3 & agggagctaccc $\underline{\text { ctg }}$ & $57-71$ \\
ODNAs4 & tcacatctgagaatgg & $2245-2260$ \\
\hline
\end{tabular}

conjugated to horseradish peroxidase followed by 3-amino9 -ethylcarbazole (Dako). The specific location of the $\alpha$-actin in the media of aorta was used to define the medial boundaries. The media thickness was then measured at a magnification of X40 in slides counterstained with Haematoxylin (Dako).

\section{RESULTS}

\section{Cloning of full-length rat 6A3-5 CDNA}

The cloned gene has a $6569 \mathrm{bp}$ cDNA sequence (GenBank accession number: AJ440711) and a deduced amino acid sequence corresponding to a $5276 \mathrm{bp}$ open-reading frame (Figure 1(a)). The cDNA contained $1268 \mathrm{bp}$ in the $3^{\prime}-$ untranslated region; the $5^{\prime}$-untranslated sequence is not totally cloned. The putative 1758 amino acid 6A3-5 protein has an expected molecular weight of $180 \mathrm{kDa}$ and bears four conserved motifs (Figure 1(b)). The first motif is a DNA binding domain, called AT-rich interaction domain or ARID, located in the N-terminal half (aa 568 to 672). Two other motifs comprising evolutionary conserved domains known as OHD (Osa Homology Domain)-1 (aa 1114 to 1200) and OHD2 (aa 1437 to 1758 ) are present within the $C$-terminal half of the protein. These three motifs are the signature of a novel family of transcription modulators called Osa family and indicate that $6 \mathrm{~A} 3-5$ is the rat Osa2 homologue. Finally, a fourth motif represented by a nuclear localization signal is also present in the $C$-terminal of $6 \mathrm{~A} 3-5 / \mathrm{Osa} 2$ sequence suggesting a nuclear localization of this protein that was subsequently confirmed.

\section{Multiple sequence alignment and homologies to rat 6A3-5/Osa2}

Protein similarity searches revealed two subgroups with significant homologies to rat $6 \mathrm{~A} 3-5 / \mathrm{Osa} 2$ protein. The 1st subgroup comprises proteins bearing ARID, OHD1, and OHD2 functional domains. This group shows a remarkably high degree of conservation of amino acid sequences, and includes the recently cloned human Osa2 [14]. This protein appears to be the human orthologue of rat $6 \mathrm{~A} 3-5 / \mathrm{Osa} 2$, mouse, and human Osa1 [17], Drosophila Osa/eyelid [18] and yeast SWI1 protein [19] (Figure 2). The 2nd subgroup shows homologies that are limited to the ARID domain and include Drosophila dead-ringer protein [20], its homologues in mouse (bright) [21] and human (DRIL-1), mouse Mrf2 [22], and the murine and human jumonji proteins (Table 2).

\section{Cellular localization}

VSMC characterized with anti- $\alpha$-actin antibody showed its nucleus to be equally labelled with an anti 6A3-5/Osa2 [14] or an anti-NFKB antibody. Negative controls showed no labelling (Figure 3).

\section{A3-5 expression in different phenotype of vascular SMC}

Transcription levels of 6A3-5/Osa2 and $\alpha$-actin markers were measured after dedifferentiation of ex vivo SMCs from a contractile (passage $0, \mathrm{P} 0$ ) to an in vitro synthetic phenotype (passage 9, P9). Northern-blots showed that 6A3-5 is upregulated by 3 -fold $(n=3)$ in the synthetic phenotype in comparison to the contractile quiescent phenotype. In contrast, $\alpha$-actin expression is present in the contractile SMCs phenotype and lost on differentiation to a synthetic phenotype (Figure 4(a) and data not shown) [23]. The 6A3$5 /$ Osa2 gene was significantly upregulated in a smooth muscle cell line (V8) that was observed to be highly proliferating [24] compared with secretory/ synthetic cells (results not shown).

\section{Time course and dose effect of PDGF on 6A3-5/Osa2 in VSMC}

Human and rat (data not shown) VSMC were serum starved, inducing a down-regulation of 6A3-5/Osa2 mRNA expression levels, and then treated with $20 \mathrm{ng} / \mathrm{ml}$ of PDGF-BB for $0,2,4,8$, and 24 hours. Northern blot analysis showed that the levels of 6A3-5/Osa2 mRNA reached a peak at 2 hours and remained above the control level for at least 24 hours after PDGF treatment (Figure 5(a)). In addition, a PDGF dosedependant effect was also observed with a maximal increase achieved at $20 \mathrm{ng} / \mathrm{ml}$ (Figure 5(b)). Similar results were observed at 4 hours, by Western blot, for 6A3-5/Osa2-protein expression (Figures 5(c), 5(d)).

\section{Antisense ODN inhibition of 6A3-5/Osa2 expression and VSMC proliferation}

A series of 20-base phosphorothioate antisense ODN (Table 1, ODN $\mathrm{OS1}_{\mathrm{AS}-4}$ ) was screened for its ability to selectively inhibit 6A3-5/Osa2 protein expression in human VSMC. After transfection, VSMC were stimulated by PDGF-BB for 4 hours. The $\mathrm{ODN}_{\mathrm{AS} 3}$, which hybridizes to the 6A35/Osa2 ATG translation initiation site, showed a significant inhibition of 6A3-5/Osa2 mRNA and protein expression in comparison to its sense, scrambled, and mismatched controls (Figures 6(a), 6(b), 6(c)). Moreover, treatment of human VSMC with increasing concentrations of $\mathrm{ODN}_{\mathrm{AS} 3}$ 
1 agccccggca cccctggacc gaccatgggc agatcccagg gcagtccgat ggacccaatg 61 gtgatgaaga gacctcagtt gtatgggatg ggcactcacc cccattcgca gccgcagcag 121 agcagcccat acccaggagg tgcctacggc cccccaggcg cacagcggta tccccttggc 181 atgcagggcc gggctccagg ggccctggga ggcttgcagt acccacagca gcagatgcca 241 cctcagtatg gacagcaagg tgtgagtggt tactgccagc agggccaaca gccatattac 301 agccagcagc cgcagccccc gcacctccca ccccaggcgc agtatctgcc gtcccagtcc 361 cagcagaggt accagccgca gcaggacatg tctcaggaag gctatggaac tagatctcaa 421 cctcctctgg cccccggaaa acctaaccat gaagacttga acttaataca gcaagaaaga 481 ccatcaagtt taccagatct gtctggctcc attgatgacc tccccacggg aacggaagca 541 actttgagct cagcagtcag tgcatccggg tccacgagca gccaagggga tcagagcaac 601 ccggcgcagt cgccttctc cccacatgca tcccctcatc tctccagcat cccggggggc 661 ccatctccct ctcctgttgg ctctcctgta ggaagcaacc agtctcgatc tggcccaatc 721 tctcctgcaa gtatcccagg ttttatggca ggcacacaaa gaaccctca gatggctcag 781 tatggacctc aacagacagg accatccatg tcgcctcatc cttctcctgg gggccagatg 841 catgctggaa tcagtcgctt tcagcagagt aactcaagtg ggacttacgg tccacagatg 901 agccagtatg gaccacaagg taactactcc agacccccag cgtatagtgg ggtgcccagt 961 gcaagctaca gcggcccagg gcccggtatg ggtatcagtg ccaacaacca gatgcatgga 1021 caagggccaa gccagccatg tggtgctgtg cccctgggac gaatgccatc agctgggatg 1081 cagaacagac catttcctgg aatatgcgc agcatgcccc ccagttctcc tggcatgtct 1141 cagcagggag ggccaggaat gggccgggca ccaggcccac caatgcccac tgtgaaccgc 1201 aaggcccagg aagctgccgc ggctgtgatg caggctgctg caaactcagc ccaaagcagg 1261 caaggcagct ttcctggcat gcaccagagt ggactcgtgg cctccagctc tccctacagc 1321 cagcccatga acaacaactc caacctaatg ggcacacagg cccagcccta cagcatcaca 1381 cccaccatgg tgaacagctc tacagcatct atgggtctta cagatatgat gtctcccagt 1441 gtgtccaaac tgtccgtgcc tctcaaagca gatggcaaag aagaaggtgt gccccagccc 1501 gagagcaagt caaaggatag ctacagctct cagggtattt ctcagcctcc aactccaggc 1561 aacctgccag tcccttcccc aatgtccccc agctctgcca gcatctcctc ctttcacgga 1621 gatgagagtg acagcattag cagcccaggc tggcccaaga ctccatcaag ccccaagtcc 1681 agctcctctt ccaccactgg ggagaagatc acaaaagtgt acgagctggg gactgcgccg 1741 gagaggaagc tgtgggtcga ccgctacctc acattcatgg aggagagggg gtcccccgtg 1801 tccagtctgc cggcagtggg caagaagccc ctggacctgt tccgactcta tgtgtgcgtc 1861 aaagagatcg gaggcttggc gcaggttcat acaaacaaga agtggcgcga gctggcaacc 1921 aacccgaacg ttggcacttc gagcagcgca gccagctccc cgaagaagca atatattcag 1981 tacctgttcg ccttcgagtg caaatcgag cgtggggagg agcccccgcc ggaagtcttc 2041 agcacggggg atgcgaagaa gcagcccaag ctccagccgc catctcctgc caactcggga 2101 tccttacaag gtccacagac gccacagtca actggcagca gttccatggc agaggttccc 2161 ggcgacccga agccaccaac cccagcctcc accctcacg gacaggggac ccccatgcaa 2221 agcggaagaa gcagtacagt cagtgtgcac gacccgttct cagacgtgag tgattcagcg 2281 tacccaaac ggaactccac gactccaaac gccccatacc agcagggcat gggcatgcca 2341 gacatgttgg gcaggatgcc ctatgcgccc aacaaggacc ctttcagtgg aacgagaaaa 2401 gtgcctggaa gcagcgagcc ctttatgaca caaggacaga tgcccaacag cagtatgcag 2461 gacatgtaca accagagtcc ctcaggtgcc atgtccaatc tgggcatggg acagcggcag 2521 caatttccct atggaaccag ttacgaccga aggcacgagg cttacgggca gcagtaccca 2581 ggccaaggcc ctcccacagg acagccaccg tatggaggac accagcctgg cctgtaccca 2641 cagcagccga attacaaacg ccatatggat ggcatgtacg ggcctccagc caagcgccac 2701 gagggagaca tgtacaacat gcagtatggc agccaacagc aggagatgta caaccagtac 2761 ggaggctcct actctggccc ggacagaagg cccatccagg gacagtatcc ctaccctac 2821 aacagagaaa ggatgcaggg cccaggccag atgcaaacag atggaatccc acctcacatg 2881 atgggtggcc ccatgcagtc atcttccaat gaggggcctc agcagaatat gtgggctaca 2941 cgcaatgata tgccttatcc ctaccagaac aggcaaggcc caggtggcce tgcacaggca 3001 ccccctacc caggcatgaa ccgcacagat catatgatgg tacctgatca gaggatcaat 3061 cacgagagcc agtggcettc tcatgtcagc cagcgccagc cttatatgtc atcatcggcc 3121 tccatgcaac ccatcacgcg cccacctcag tcatcctacc agacgccgec gtcactgcca 3181 aaccacatct ccagggcacc cagccetgcc tccttcccgc gctccctgga gagccgcatg 3241 tctccaagca agtccccct cctgcctgcc atgaagatgc agaaggtcat gcccacggtc 3301 cccacatccc aggtcaccgg accaccccca cagccaccgc caatcagaag ggagattacc 3361 tttcctcctg gctcagtaga agcatcgcag ccagtcccga aacaaaggcg gaagattacc 3421 tcaaagata ttgttactcc cgaggcgtgg cgtgtgatga tgtcccttaa atccggtctg 3481 ttggctgaga gcacgtgggc tttggacacc atcaacattc tcctctatga tgacagcact 3541 gtcgccacct tcaatctttc ccagctgtcg gggttcctgg aactactagt ggagtacttc 3601 cgaaagtgcc taattgacat tttcggtatt cttatggaat atcaagtggg cgaccccagc 3661 caaagggcgc tcgatcaccg cacagggaag aaagacgaca gccagtcctc ggaggacgac 3721 tctgggaaag aagaagaaga tgccgagtgt ctggaggagg aggaggagga ggaggaaga 3781 gaggaggagg aagaggagca agtcagtaaa aagacagagt cagagggcaa gagcagctct 3841 gcctagctg ctccagacac cactgcggac cccaaggaga cgccgaggca ggccagtaag 3901 ttcgacaagc tgcccataaa gattgtcaag aagaacaacc tgtttgtggt ggaccggtcc 3961 gacaggctgg gtcgggttca agagttcaac agcgggctcc tccactggca gctgggtggt 4021 ggcgacacca ccgagcacat cctcactcac ttcgagagca agatggagat ccctcctcgc 4081 aggcgtccac ctgcgcctct cagctccacg ggtaagaaga aagagctggc aggcaagggc 4141 gattctgaag agcagccaga gaaagcatc atagccacca tcgatgatgt cctgtctgcc 4201 cgaccagggg ccctgccgga agacagcaac cccggacccc aaaccgagag cggcaagtt 4261 ccctttggaa tccagcaggc caaaagccac cggaacatca ggctcctgga ggacgagccc

(a)

FIGURE 1: Full-length ORF sequence of rat 6A3-5, 6569pb. (a) The full-length rat 6A3-5 cDNA has a first methionine that corresponds to an ATG codon (position 25 underlined) and a stop codon (TGA, position 5301) followed by polyadenylation signal (position 6299). (b) ORF translation sequence with 1758 aa. Conserved motifs are indicated: ARID motif (568-672) is underlined, OHD motifs (OHD1: 1114-1200; OHD2: 1437-1758) are boxed, and NLS (nuclear localization signal) (1352-1369) in bold. 
4321 aggagccgag acgagactcc cctgtgcacc attgcgcact ggcaggactc cctggccaaa 4381 cgctgcatct gtgtgtccaa catcgtgcgg agcttgtctt tcgtgcctgg caacgacgca 4441 gagatgtcca aacatccggg cttggtgccg atcctaggaa agtcgattct gctgcatcat 4501 gagcatccgg agagaaaacg ggcgccacag acctatgaga aggaggagga tgaggacaag 4561 ggggtggcct gcagcaaaga tgagtggtgg tgggactgcc tcgaggtctt gcgggacaac 4621 acactggtca cattggctaa catttccggg cagctagact tgtctgctta cacagagagc 4681 atctgcttgc cgatcctgga cggcttgctg cactggatgg tgtgcccgtc tgcagaggca 4741 caggacccct ttcccactgt ggggcccaac tcagttctat cgccacaaag acttgtgctg 4801 gagacactgt gtaaactcag tatccaggac aacaatgtgg acctgatctt ggccacacct 4861 ccatttagtc gtcaggagaa attttatgct acattagtta ggtacgttgg ggatcgcaaa 4921 aacccagtct gccgagaaat gtccatggcg cttttatcga accttgccca gggggataca 4981 ctggcagcga gggcaatagc tgtgcagaaa ggaagcattg ggaacttgat aggcttcctg 5041 gaggacgggg tcacgatggc gcagtatcag cagagccagc ataccctcat gcacatgcag 5101 cccccgcctc tggaaccccc cagtgtggac atgatgtgca gggcggccaa agctttgctg 5161 gctatggcca gagtggatca gaaccgctcg gagttccttt tgcacgaggg tcggttgctg 5221 gatatctcga tatccgctgt cctgaactct ctggttgcgt ctgtcatctg tgatgtactg 5281 tt cagattg ggcagttatg acacccgtga gggcacacat gtgtgaggga acattagagg 5341 gtcacatatg actggctgtt ttctgttctc gtttatccaa tgtaggaaga aggaaagaa 5401 aatctttgc ttctctgccc cattcactat ttaccaattg ggaattaaag aaatcattaa 5461 tttgaacagt tataaltaa tatttgctgt ctgtgtgtat aagtacatcc gttgggggat 5521 ttctgttct ttctctttt tttaaccaaa gttgccgtct agtgcattca caggtcacat 5581 gtttttttgt ttttttcata atttttttca tgttgtatta cagttttagg gaagtgaatt 5641 cactttataa agtaaaagg tttggcaaaa aatgctgata ggaaatttc accacactga 5701 gtcaaaaagg tgaaaggaaa aattgatcct taaattgatt tcctatgaat tttattcttc 5761 gcagaatgaa aaaagcgaaa gtgcatccca ttgcccaaag ctctgtgcaa tagaaacttc 5821 tagagatgta ggtgtagggg ctcgaggtat ggcagtcagc agtctggccc agtgatgctg 5881 ttctctccac aggaaagcgg ttgcattagg cctcgagcaa aaaaccgcac tctcagttag 5941 gggtgaaal ccactcctaa ccgccaacag caggattgct tcctcaccac gaccgccatg 6001 tctgctgcga ctcagcctcc acctcacaga tcttcgtgat tcctttcatc atttttaaa 6061 tatttttttt ttactgccta tgggctgtga tgtatataga agttgtacat taaacatacc 6121 ctcatattt tttctttct ttttttttt ttagtacaaa gttttagtt tcttttcat 6181 gatgtggtaa ctacgaagtg atggtagatt taataatt tttatttta tttatatat 6241 tttttcatta ggaccatatc tccaaaaac aagaaaaga aacaaaaat tacaaaaat 6301 taaacaaaca aaaaagggg gtaatgtaca agtttctgta tgtataaagt catgctctgt 6361 tgggagggca gctggtccca atttgcttca tgaatcaagg tgtggaaatg gttgcatacg 6421 gattgattta gaaatgaat accagtacat acaaaaaaa agaaaaaga aaaccaact 6481 aatgaagaa acacaacttc aagatttt ctgtgacaag aatccgcatt tgtatttcaa 6541 gataatgtag tttaagaaaa aaaaaaaa

(a)

1 MGRSOGSPMD PMVMKRPQLY GMGTHPHSOP QQSSPYPGGA YGPPGAQRYP LGMOGRAPGA 61 LGGLQYPQQQ MPPQYGQQGV SGYCQQGQQP YYSQQPQPPH LPPQAQYLPS QSQQRYQPQQ 121 DMSQEGYGTR SQPPLAPGKP NHEDLNLIQQ ERPSSLPDLS GSIDDLPTGT EATLSSAVSA 181 SGSTSSQGDQ SNPAQSPFSP HASPHLSSIP GGPSPSPVGS PVGSNQSRSG PISPASIPGF 241 MAGTORNPOM AOYGPOQTGP SMSPHPSPGG OMHAGISRFO QSNSSGTYGP OMSOYGPOGN 301 YSRPPAYSGV PSASYSGPGP GMGISANNQM HGQGPSQPCG AVPLGRMPSA GMQNRPFPGN 361 MRSMPPSSPG MSQQGGPGMG RAPGPPMPTV NRKAQEAAAA VMQAAANSAQ SRQGSFPGMH 421 QSGLVASSSP YSQPMNNNSN LMGTQAQPYS ITPTMVNSST ASMGLTDMMS PSVSKLSVPL 481 KADGKEEGVP QPESKSKDSY SSQGISQPPT PGNLPVPSPM SPSSASISSF HGDESDSISS 541 PGWPKTPSSP KSSSSSTTGE KITKVYELGT APERKLWVDR YLTFMEERGS PVSSLPAVGK 601 KPLDLFRLYV CVKEIGGLAQ VHTNKKWREL ATNPNVGTSS SAASSPKKQY IQYLFAFECK 661 IERGEEPPPE VFSTGDAKKO PKLQPPSPAN SGSLQGPQTP QSTGSSSMAE VPGDPKPPTP

721 ASTPHGQGTP MQSGRSSTVS VHDPFSDVSD SAYPKRNSTT PNAPYQQGMG MPDMLGRMPY

781 APNKDPFSGT RKVPGSSEPF MTQGQMPNSS MQDMYNQSPS GAMSNLGMGQ RQQFPYGTSY 841 DRRHEAYGQQ YPGQGPPTGQ PPYGGHQPGL YPQQPNYKRH MDGMYGPPAK RHEGDMYNMQ 901 YGSQQQEMYN QYGGSYSGPD RRPIQGQYPY PYNRERMQGP GQMQTDGIPP HMMGGPMQSS 961 SNEGPQQNMW ATRNDMPYPY QNRQGPGGPA QAPPYPGMNR TDHMMVPDQR INHESQWPSH 1021 VSQRQPYMSS SASMQPITRP PQSSYQTPPS LPNHISRAPS PASFPRSLES RMSPSKSPFL 1081 PAMKMQKVMP TVPTSQVTGP PPQPPPIRRE ITFPPGSVEA SQPVPKQRRK ITSKDIVTPE 1141 AWRVMMSLKS GLLAESTWAL DTINILLYDD STVATFNLSQ LSGFLELLVE YFRKCLIDIF 1201 GILMEYQVGD PSQRALDHRT GKKDDSQSSE DDSGKEEEDA ECLEEEEEEE EEEEEEEEQV 1261 SKKTESEGKS SSALAAPDTT ADPKETPRQA SKFDKLPIKI VKKNNLFVVD RSDRLGRVQE 1321 FNSGLLHWQL GGGDTTEHIL THFESKMEIP PRRRPPAPLS STGKKKELAG KGDSEEQPEK 1381 SIIATIDDVL SARPGALPED SNPGPQTESG KFPFGIQQAK SHRNIRLLED EPRSRDETPL 1441 ETIAHWQDSL AKRCICVSNI VRSLSFVPGN DAEMSKHPGL VPILGKSILL HHEHPERKRA 1501 PQTYEKEEDE DKGVACSKDE WWWDCLEVLR DNTLVTLANI SGOLDLSAYT ESICLPILDG

1561 LLHWMVCPSA EAQDPFPTVG PNSVLSPQRL VLETLCKLSI QDNNVDLILA TPPFSRQEKF

1621 YATLVRYVGD RKNPVCREMS MALLSNLAQG DTLAARAIAV QKGSIGNLIG FLEDGVTMAQ

1681 QQQSQHTLMH MQPPPLEPPS VDMMCRAAKA LLAMARVDQN RSEFLLHEGR LLDISISAVL

1741 NISLVASVICD VLFQIGQL

ARID

OHD1

(b)

Figure 1: Continued. 


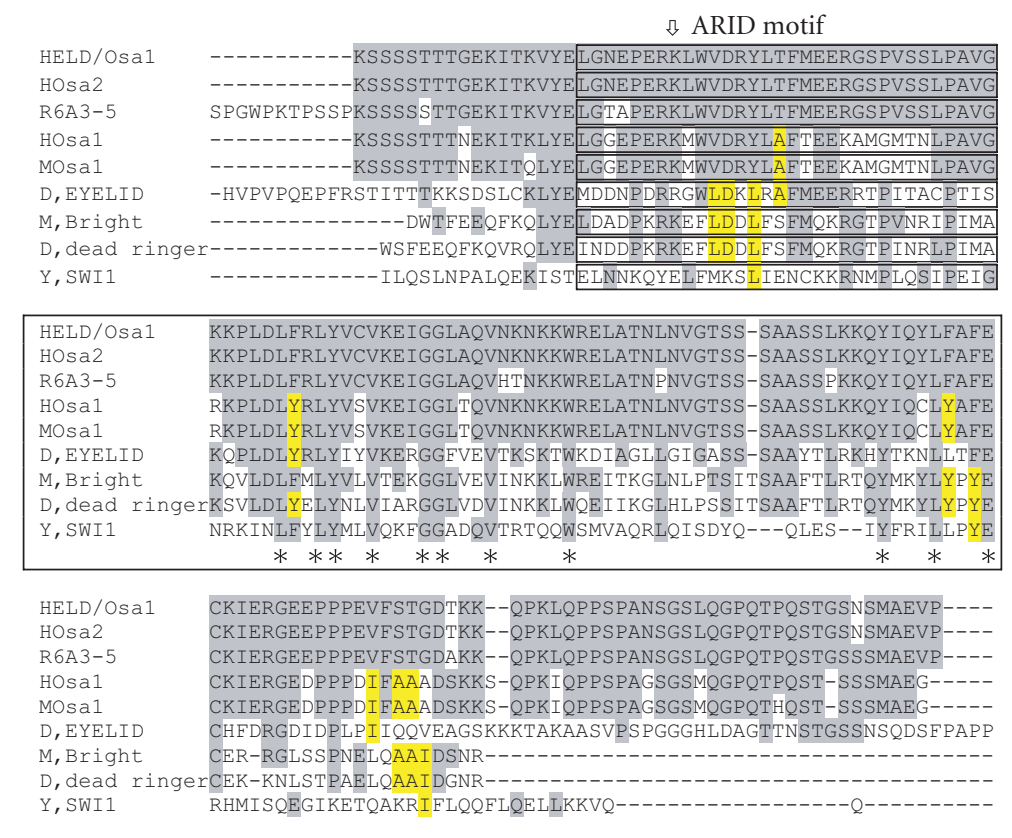

1140

HELD/Osa1 KKPLDLFRLYVCVKEIGGLAOVNKNKKWRELATNLNVGTSS-SAASSLKKOYIQYLEAFE $\begin{array}{ll}\text { HOSa2 } & \text { KKPLDLFRLYVCVKE IGGLAQVNKNKKWRELATNLNVGTSS-SAASSLKKQYIQYLFAFE } \\ \text { R6A3-5 } & \text { KKPLDLFRLYVCVKEIGGLAQVHTNKKWRELATNPNVGTSS-SAASSPKKQYIQYLFAFE }\end{array}$ HOSa1 RKPLDLYRLYVSVKEIGGLTQVNKNKKWRELATNLNVGTSS-SAASSLKKQYIQCLYAFE RKPLDLYRLYVSVKEIGGLTQVNKNKKWRELATNLNVGISS-SAASSLKKQYIQCLYAF KOPLDLYRLYIYVKERGGEVEVTKSKTWKDIAGLLGIGASS-SAAYTLRKHYTKNLLTE KOVLDLFMLYVLVTEKGGLVEVINKKLWREITKGINIPTSITSAAFTLRTOYMKYLYPY D, dead ringerKSVLDLYELYNLVIARGGLVDVINKKLWQEI IKGLHLPSSITSAAFTLRTQYMKYLYPYE

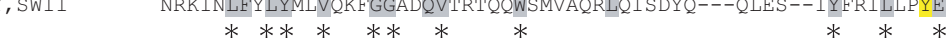

(a)

ת OHD1

\begin{tabular}{|c|c|}
\hline HELD/Osal & PPGSVEASQPVLKQRRKITSKDIVTPEAWRVMMSLKSGLLAESTWALDT INILLYDDSTV \\
\hline HOsa2 & PPGSVEASQPVLKQRRKITSKDIVTPEAWRVMMSLKSGLLAESTWALDT INILLYDDSTV \\
\hline R6A3-5 & PPGSVEASQPVPKQRRKITSKDIVTPEAWRVMMSLKSGLLAESTWALDT INILLYDDSTV \\
\hline Hosa1 & PPGSVEATQPVLKQRRRLTMKDIGTPEAWRVMMSLKSGLLAESTWALDT IN I LLYDDNS I \\
\hline Mosa1 & PPGSVEATQPVLKQRRRLTMKDIGTPEAWRVMMSLKSGLLAESTWALDT IN I LLY DDNS I \\
\hline D, EYELID & PHDSVESTTPVLYRRKRLTKADVCPVDPWRI FMAMRSGLLTECTWALDVLNVLLFDDSTV \\
\hline M, Bright & 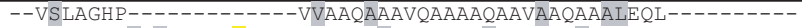 \\
\hline \multirow{2}{*}{\multicolumn{2}{|c|}{$\begin{array}{l}\text { D, dead ringerQQQRQRSQSPDLSKHEALS----AQVALWHMY HNNNSPPGSAHTSPQQRE--------- } \\
\text { Y, SWI1 } \\
\text { NYISKIGEKIDSNKPIFLFAPELGAINLHALSMSLQSKNLGEINTALNTLLVTSADSN- }\end{array}$}} \\
\hline & \\
\hline HELD/Osal & ATFNLSQLSGFLELLVEYFRKCLIDIFGILMEYEVGDPSQKALDHNAARKDDSQSLANDS \\
\hline HOsa2 & ATFNLSQLSGFLELLVEYFRKCLIDIFGILMEYEVGDPSQKALDHNAARKDDSQSLADDS \\
\hline R6A3-5 & ATFNLSQLSGFLELLVEYFRKCLIDIEGI LMEYQVGDPSQRALDHRTGKKDDSQSSEDDS \\
\hline Hosa1 & MTFNLSQLPGLLELLVEYFRRCLIEIFGILKEYEVGDPGQRTLLD-PGRFSKVSSPAPME \\
\hline Mosa1 & MTFNLSQLPGLLELLVEYFRRCLIEIFGILKEYEVGDPGQRTLLD-PGRFTKVSSPAHTE \\
\hline D, EYEI & QFFGISNLPGLLTLLLEHFQKNLAEMFDERENEEQSALLAEDADDDADSGTVMCEKLRTS \\
\hline M, Bright & \\
\hline \multicolumn{2}{|c|}{ D, dead ringer-ALNLSDS---------- } \\
\hline Y, SWI1 & LKISLVKYPELIDSLAILG----MNLLSNLSQNVVPYHRNTSDYYYEDAGSNQYYVTQHD \\
\hline
\end{tabular}

(b)

FIgURe 2: Alignments of conserved domains of ARID proteins by CLUSTALW program. (a) ARID motif alignment among different species is shown. (b) OHD-1 (Osa hoomology domain-1) motif alignment. (c) OHD-2 (Osa hoomology domain-1) motif alignment. Conserved aa residues are shown by underlining. Identical residues are indicated in grey. H: human, r: rat, M: Mouse, d: Drosophila, Y: yeast (hELD/OSA1 or hOsa2: human homolog of 6A3-5/Osa2).

$(25,100,200 \mathrm{nM})$ resulted in a dose-dependent reduction in $6 \mathrm{~A} 3-5 /$ Osa2 protein level (Figure $6(\mathrm{~d})$ ) but had no effect on P53 expression. Indeed, 6A3-5/Osa2 expression is reduced by $60-70 \%$ in presence of $200 \mathrm{nM}$ of antisense $\mathrm{ODN}_{\mathrm{AS} 3}$.

To investigate whether reduction of 6A3-5/Osa2 expression affected PDGF-induced proliferation, serum starved human VSMC were exposed to $\mathrm{ODN}_{\mathrm{AS} 3}$ and then stimulated by PDGF-BB for 24 hours. ODN ${ }_{\mathrm{AS}}$ reduced by $50-60 \%$ PDGF-induced proliferation in human VSMC (Figure 7(a)) while sense, scrambled, or mismatched oligonucleotides derived from $\mathrm{ODN}_{\mathrm{AS} 3}$ had no effect. Moreover, increasing the concentration of $\mathrm{ODN}_{\mathrm{AS} 3}$ significantly reduced PDGFinduced proliferation of VSMC in a dose-dependent manner (Figure 7(b)).

\section{Expression of 6A3-5/Osa2 and vascular Phenotype in $L H$ versus $L L$ rats}

Quantitative PCR performed on aorta excised from hypertensive (LH) rats exhibited significantly increased 6A3-5/Osa2 gene expression levels compared to those present in normotensive (LL) rats (Figure 8(a)). A significant decrease in VSMC contractile markers, calponin and 
$\sqrt{1} \mathrm{OHD} 2$

HELD/OSA1 KFPFGIQQAKS--------------- HRNIKLLEDEPRSRDETPLCTIAHWQDSLA

HOsa2 KFPFGIQQAKS----------------HRNIKLLEDEPRSRD ETPLCTIAHWQDSLA

R6A3-5 KFPFGIQQAKS----------------HRNIRLLEDEPRSRD ETPLCTIAHWQDSLA

HOsa1 KFPFGISPAQS----------- HRNIKILEDEPHSKD ETPLCTLLDWQDSLA

MOsal KFPFGISPAQS---------------HRNIKILEDDPHSKDETPLCTLLDWQDPLA

D, EYELID RLTNGVAPCSSTPAIFDPRTTAKDEARVLQRRRDSSFEDECYTRDEASLHLVSESQDSLA

M, Bright -

$D$, dead ringerDYAHG--_-_-_-_-_-_-_-_-_-_-_-_-_---EHNTTGNSSSMHDDSEPOOMNG Y, SWI1 ILNFKKDLVIVLS-------------NISHLLEIASS I DCLIILILVISFGQPKL

HELD/Osa1 KRCICVSNIVRSLSFVPGNDAEMSKHPGL-VLILGKLILLHHEHPERKRAPQTYEKEEDE

$\begin{array}{ll}\text { HELD/OSa1 } & \text { KRCICVSNIVRSLSFVPGNDAEMSKHPGL-VLILGKLILLHHEHPERKRAPQTYEKEEDE } \\ \text { HOsa2 } & \text { KRCICVSNIVRSLSFVPGNDAEMSKHPGL-VLILGKLILLHHEHPERKRAPQTYEKEEDE }\end{array}$

R6A3-5 KRCICVSNIVRSLSFVPGNDAEMSKHPGL-VPILGKSILLHHEHPERKRAPQTYEKEEDE

HOsa1 KRCVCVSNTIRSLSFVPGNDFEMSKHPGL-LLILGKLILLHHKHPERKQAPLTYEKEEEQ

MOsa1 KRCVCVSNTIRSLSFVPGNDFEMSKHPGL-LLILGKLILLHHKHPERKQAPLTYEKEEEQ

D, EYELID RRCIALSNIFRNLTFVPGNETVLAKSTRF-LAVLGRLLLLNHEHLRRTPKTRNYDREEDT

M, Bright QRAVQQS-FLAMTAQLPMN-------------IR----INSQASESRQDSAVSLTSANG

D, dead ringerHHHHQTHHLDKSDDSAIENSPTTSTTTG------GSVGHRHSSPVSTKKKGGAKPQSGGK Y, SWI1 NPMASSSSSFGSESLTFNEFQLQWGKYYQTFGVDILAKLFSLEKPNLNYFKSILLNKNTGNN

\begin{tabular}{ll|}
\hline HELD/Osa1 & DKGVACS----KDEWWWDCLEVLRDNTLVTLANISGQLDLSAYTES ICLPILDGLLHWMV \\
HOsa2 & DKGVACS----KDEWWWDCLEVLRDNTLVTLANISGQLDLSAYTES ICLPIIDGLLHWMV \\
R6A3-5 & DKGVACS----KDEWWWDCLEVLRDNTLVTLANISGQLDLSAYTES ICLPIIDGLLHWMV \\
HOsa1 & DQGVSCN----KVEWWWDCLEMLRENTLVTLANISGQLDLSPYPES ICLPVLDGLLHWAV \\
MOsa1 & DQGVSCD----KVEWWWCLEMLRENTLVTLANISGQLDLSPYPES ICLPVLDGLHWAV \\
D, EYELID & DESDSCSSLQGEREWWWDYLITIRENMLVAMANIAGHLELSRYDELIARPLIDGLLHWAV \\
\hline M, Bright & SNSISMS----------VEMNGIVYTGVLFAQPP------------------PPT \\
D, dead ringerDLPTEDK---------DASSSGKLNPLETLSLLSG--------------MQFQV
\end{tabular}

$D$, dead ringerDLPTEDK---------DASSSGKLNPLETLSLLSG----------------MQFQV

Y, SWI1 LYDRNSN------NNHKDKKLLRRLLNLYNDNNKNNNNRHNLLNDVVSFLFSAIPLQQVL

\begin{tabular}{|c|c|}
\hline HELD/Osa1 & CPSAEAQDPFPTVGPNSVLSPQRLVLETLCKLSIQDNNVDLILATPPFSRQEKFYATLVR \\
\hline HOsa 2 & CPSAEAQDPFPTVGPNSVLSPQRLVLETLCKLSIQDNNVDLILATPPFSRQEKFYATLVR \\
\hline R6A3-5 & CPSAEAQDPFPTVGPNSVLSPQRLVLETLCKLSIQDNNVDLILATPPFSRQEKFYATLVR \\
\hline HOsa1 & CPSAEAQDPFSTLGPNAVLSPQRLVLETLSKLSIQDNDVDLILATPPFSHLEKLYSTMVR \\
\hline MOsa1 & CPSAEAQDPFSTLGPNAVLSPQRLVLETLSKLSIQDNNVDLILATPPFSRLEKLYSTMVR \\
\hline D, EYELID & CPSAHGQDPFPSCGPNSVLSPQRLALEALCKLCVTDANVDLVIATPPFSRLEKLCAVLTR \\
\hline M, Bright & APSAPGKGGVSS I GTN -------------------------- TTT G -- SR ---------- \\
\hline
\end{tabular}

Y, SWI1 SQSADPSLLIDQFSPVISQSLTSILVIVQKILPLSNEVEEISENNSDSNSNNN-------

\begin{tabular}{ll|}
\hline HELD/Osa1 & YVGDRKNPVCREMSMALLSNLAQGDALAARAIAVQKGS IGNLISFLEDGVTMAQYQQSQH \\
HOsa2 & YVGDRKNPVCREMSMALLSNLAQGDALAARAIAVQKGS IGNLISFLEDGVTMAQYQQSQH \\
R6A3-5 & YVGDRKNPVCREMSMALLSNLAQGDTLAARAIAVQKGS IGNLIGFLEDGVTMAQYQQSQH \\
HOsa1 & FLSDRENPVCREMAVVLLANLAQGDSLAARAIAVQKGS IGNLLGFLEDSLAATQFQQSQA \\
MOsa1 & FLSDRKNPVCREMAVVLLANLAQGDSLAARAIAVQKGS IGNLLGFLEDSLAATQFQQSQA \\
D, EYELID & HLCRNEDQVLREFSVNLLHYLAAADSAMARTVALQS PCISYLVAFIEQAEQTALGVANQH \\
\hline M, Bright & ------ TG----------ASGSTVSGGQVGLPGVSTPTMSSTSNNSLP-------- \\
D, dead ringer-----------------SETRTSSPCHAEAPTVEEEKDEEEEEEEEPKAAEEES \\
Y, SWI1
\end{tabular}

\begin{tabular}{|c|c|}
\hline HELD/Osal & NLMHMQ - PPPLEPPSVDMMCRAAKALLAMARVDENRSEFLLHEGRLLDISISAVLNSLVA \\
\hline HOsa 2 & NLMHMQ - PPPLEPPSVDMMCRAAKALLAMARVDENRSEFLLHEGRLLDIS ISAVLNSLVA \\
\hline $\mathrm{R} 6 \mathrm{~A} 3-5$ & TLMHMQ - PPPLEPPSVDMMCRAAKALLAMARVDQNRSEFLLHEGRLLDIS ISAVLNSLVA \\
\hline Hosal & SLLHMQ-NPPFEPTSVDMMRRAARALLALAKVDENHSEFTLYESRLLDISVSPLMNSLVS \\
\hline Mosal & SLLHMQ-NPPFEPTSVDMMRRAPRALLALAKVDENHSEFTLYESRLLDISVSPLMNSLVS \\
\hline D, EYELID & GINYLRENPDSMGTSLDMLRRAAGTLLHLAKHP DNRS LFMQQEQRLLGLVMSH I LDQQVA \\
\hline M, Bright & 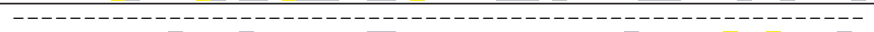 \\
\hline D, dead rin & CHRSPVK----QENEDADQDMEGSEVLLNGGASAVGG--AGAGVG--VGVGVPLLKDAVVS \\
\hline Y, SWI1 & LPS INISCVQLIKCLVEKS ICFENCLNNDPEILKKIAS I PNLFPTDLEIFQLFTNPSVDI \\
\hline HELD/Osa1 & SVICDVLFQIGQL- \\
\hline Hosa2 & SVICDVLFQIGQI \\
\hline $\mathrm{R} 6 \mathrm{~A} 3-5$ & SVICDVLFQIGQL \\
\hline Hosal & QVICDVLFLIGQS ------------------------------------------- \\
\hline Mosa1 & QVICDVLFLIGQS ----------------------------------------------- \\
\hline D, EYELID & LIISRVLYQVSRGTGPIHSVEFRLLQQRQQQQLRPGPAGKQAASAGGSATVKAETASTET \\
\hline M, Bright & ----------------------------------------------------------------- \\
\hline D, dead ri & 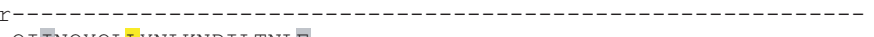 \\
\hline Y, SWI1 & QI INQYQLLYNLKNDILTNLE-------------------- \\
\hline
\end{tabular}

(c)

FIgURE 2: Continued. 


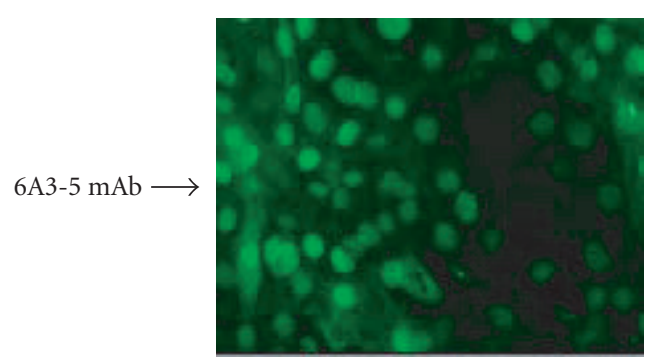

(a)

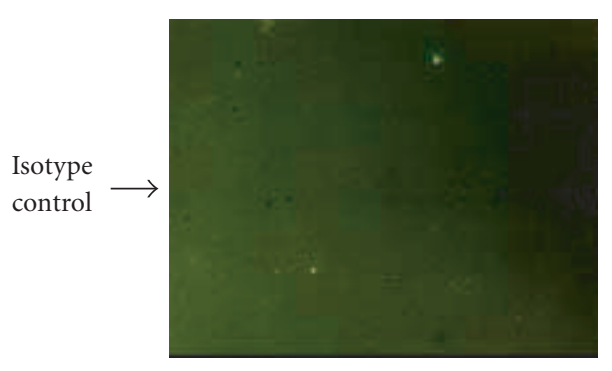

(c)

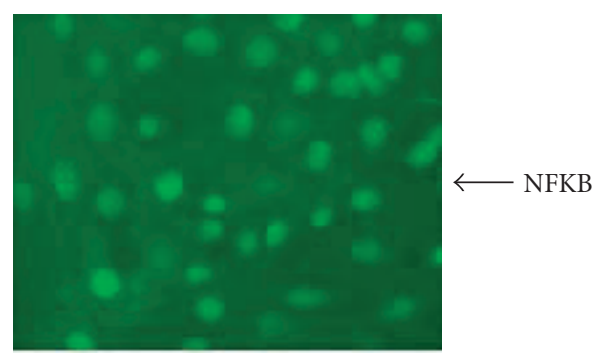

(b)

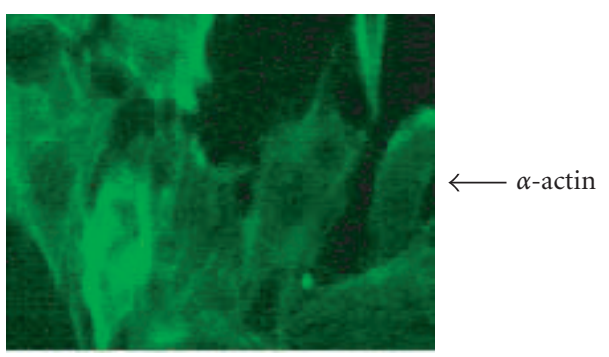

(d)

FIgure 3: 6A3-5/Osa2 cellular localization on VSMC. Actin was used as a cytoplasmic control of smooth muscle cells marker, while NFKB served as a nuclear control. Cell nucleus was labelled with an anti-6A3-5/Osa2 or an anti-NFKB antibody in comparison to isotype control (mouse IgG).

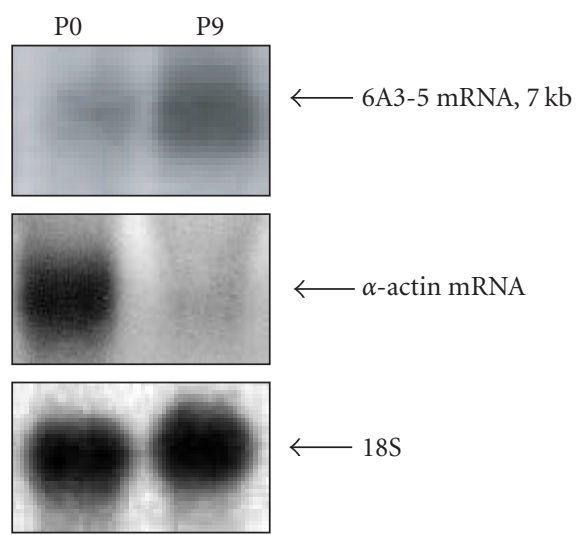

(a)

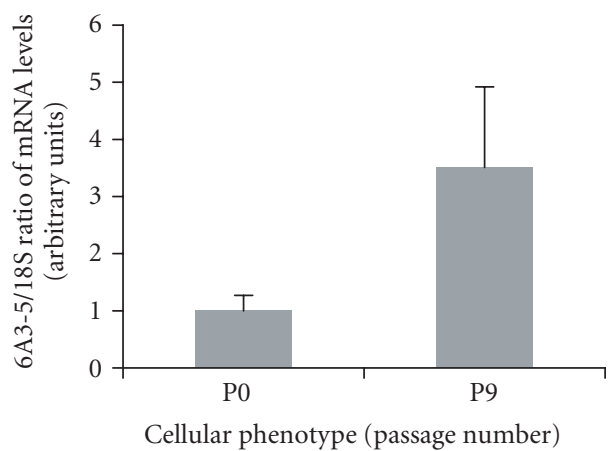

(b)

FIGURE 4: 6A3-5 and $\alpha$-actin SMC expression in different vascular SMC. (a) Levels of 6A3-5 gene transcription were compared, by northern blot, between the contractile (passage 0, P0) and the secretory/synthetic phenotype (9th passage, P9). Phenotypes were characterized by the $\alpha$-actin SMC marker. The $18 \mathrm{~S}$ served as a control for loading and quantification. (b) Quantification of 6A3-5 signals, done on 3 independent northern blots, reported to the $18 \mathrm{~S}$ levels. Results show 6A3-5 mRNA levels to be increased by 3 folds in synthetic cells compared to contractile cells.

SM22-alpha, was observed in LH but not LL rats (Figures 8(b), 8(c)).

Immunolabelling indicated the presence of 6A3-5/Osa2 in VSMC of LH and LL aortas (Figures 9(a), 9(b)), but no labelling was observed in negative controls (Figure 9(e)). Interestingly, the 6A3-5/Osa2 antibody shows similar labelling to those observed with proto-oncogene c-fos (Figures 9(c), $9(\mathrm{~d}))$. Such an increased level of $6 \mathrm{~A} 3-5 / \mathrm{Osa} 2$ was associated with a state of hypertension. Indeed, work by Aguilar et al [16] has shown that LH rats have a systolic blood pressure $(\mathrm{SBP})$ of $166 \pm 3.59$ compared to $131 \pm 2.78 \mathrm{mmHg}$ for LL. 

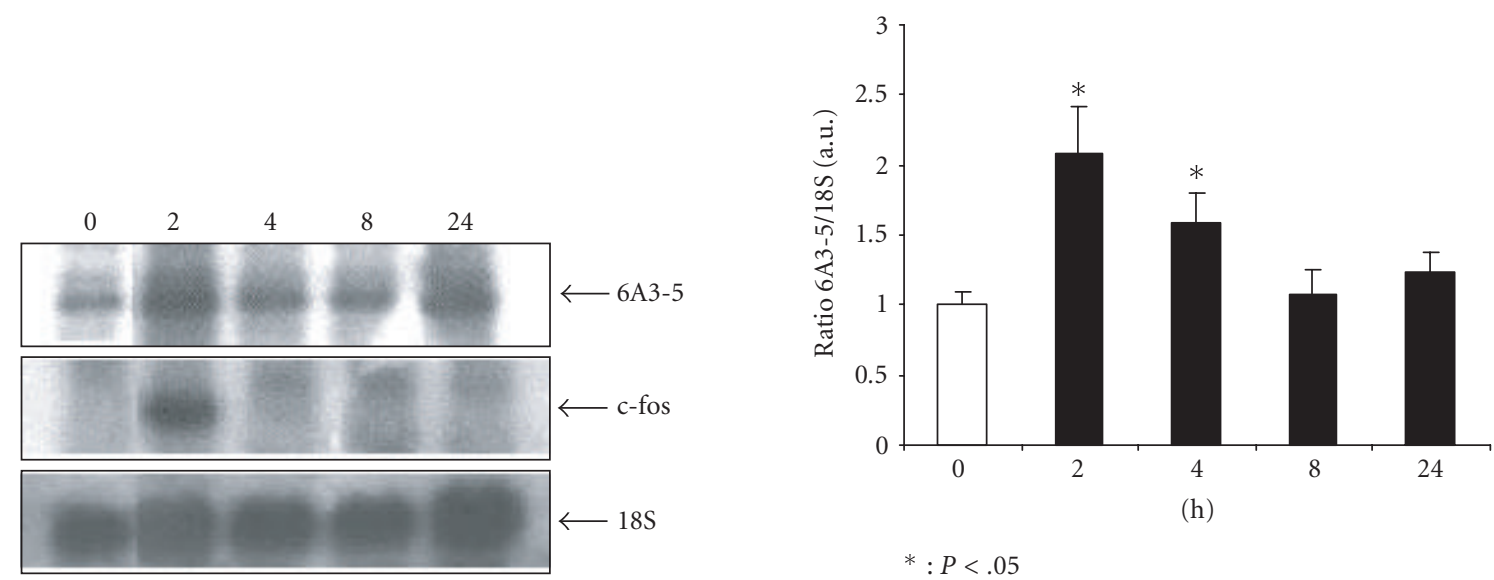

(h)

$$
*: P<.05
$$

(a)
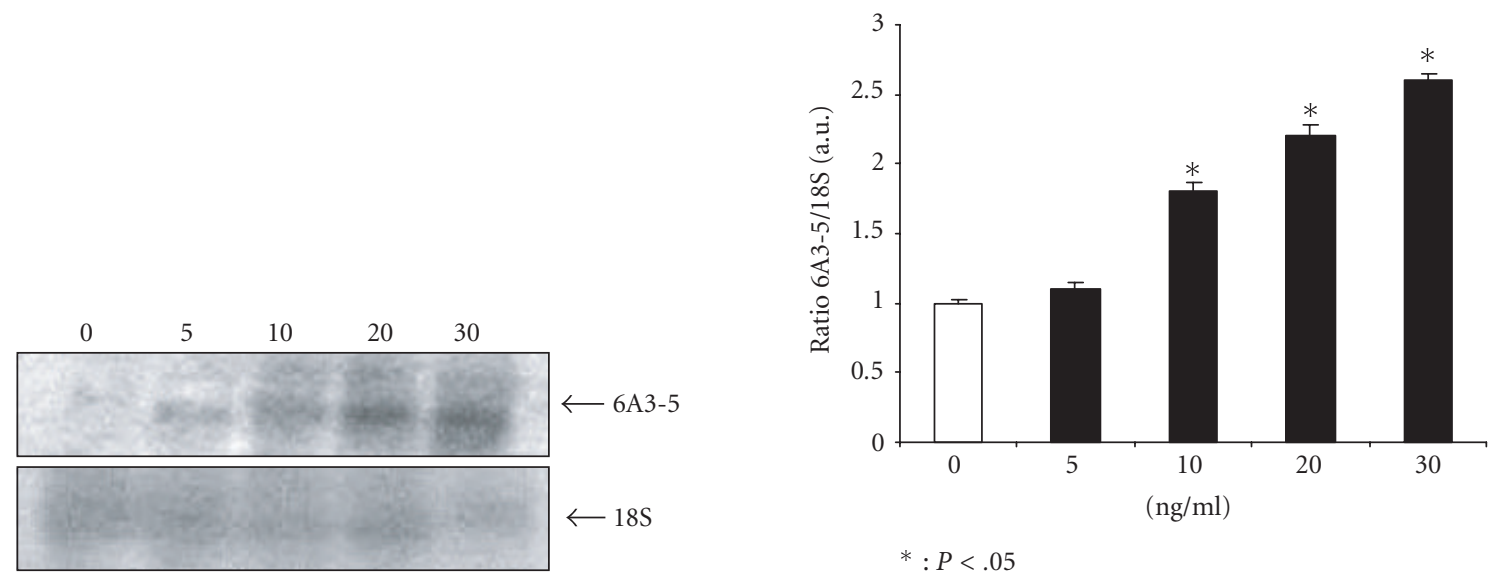

$*: P<.05$

(b)

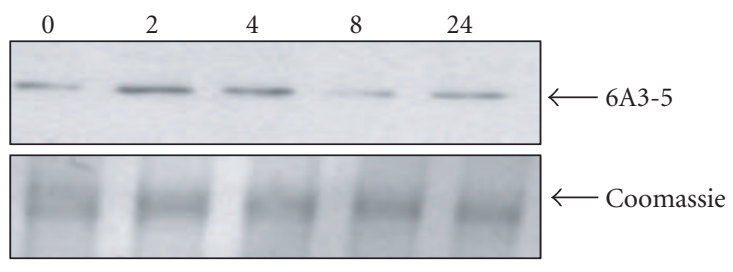

(c)

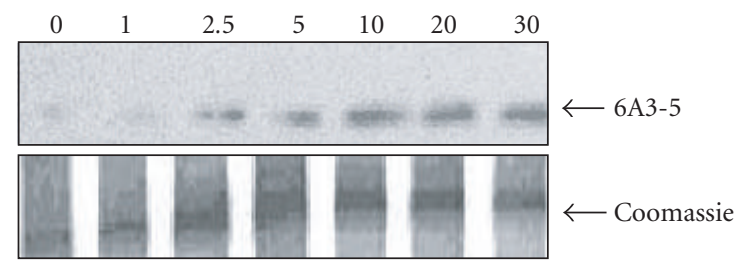

(d)

FIGURE 5: Time course and dose effect of PDGF on 6A3-5/Osa2 expression. (a) Time course of 6A3-5/Osa2 mRNA level analysed by Northernblot, following treatment of human proliferating VSMC (9th passage) with $20 \mathrm{ng} / \mathrm{ml}$ PDGF. VSMC were serum starved for 48 hours before analysis. (b) Dose effect of 6A3-5/Osa2 mRNA level analysed by Northern-blot, following treatment of VSMC with increasing concentrations of PDGF for 2 hours. (c) Time course of 6A3-5/Osa2 protein levels investigated by Western blot. (d) Dose effect of 6A3-5/Osa2 protein levels investigated by Western blot, following VSMC treatment with increasing concentrations of PDGF for 4 hours. The results are representative of three independent experiments. Northern-blots were quantified by Quantity One tool (Bio-Rad) and normalized by $18 \mathrm{~S}$ rRNA level. Data are presented as means \pm SEM. ${ }^{*}: P<.05$ versus nonstimulated control cells. The Coomassie blue-stained gel indicates equal protein loading.

\section{Expression of 6A3-5/Osa2 and vascular phenotype in ACE inhibitor treated LH and LL rats}

Four-week treatment with Perindopril (an ACE inhibitor) significantly reduced SBP in both LH (from $166 \pm 3.59$ to $134 \pm 1.84 \mathrm{mmHg}$ ) and $\mathrm{LL}$ (from $131 \pm 2.78$ to $104 \pm$ $2.39 \mathrm{mmHg}$ ) compared to untreated animals [16]. Interestingly, the 6A3-5/Osa2-gene expression level decreased in treated LH, but not LL, rats (Figure 10(a)). Moreover, VSMC contractile markers showed, by Q-PCR, a decrease 


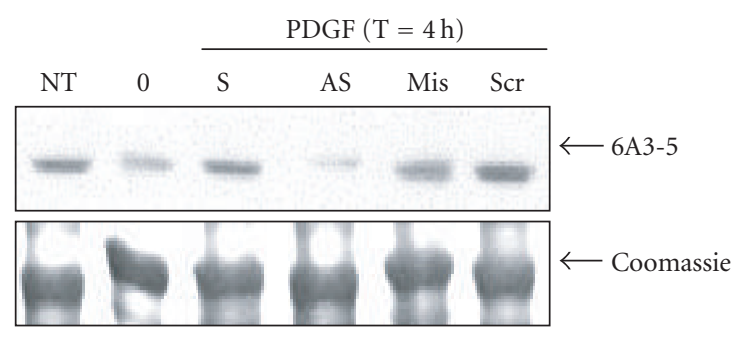

(a)

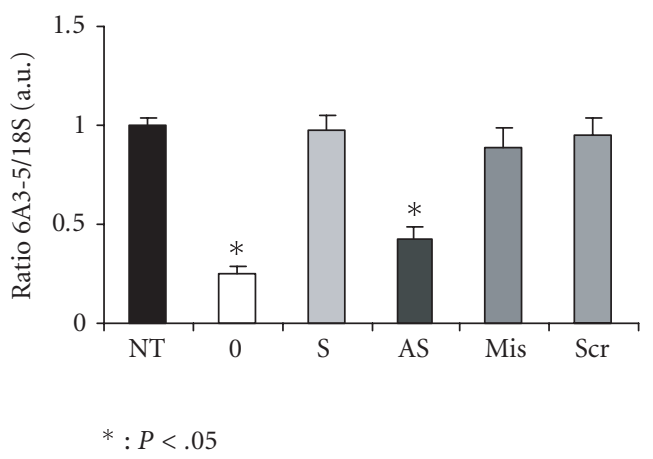

(c)

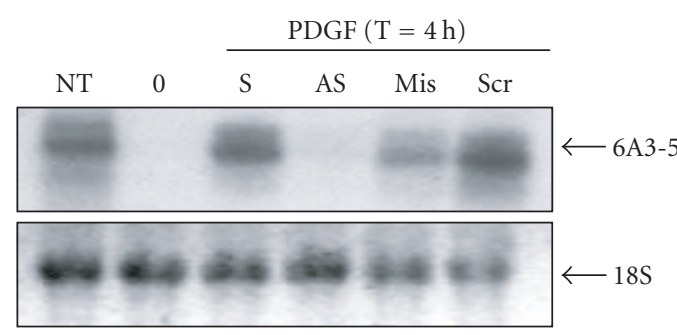

(b)

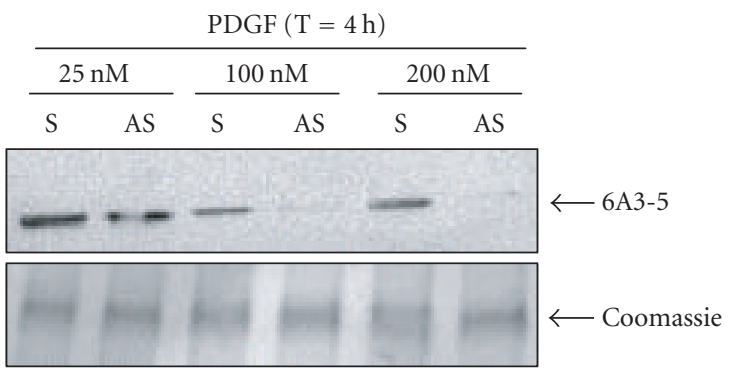

(d)

FIGURE 6: Inhibition of 6A3-5/Osa2 expression by ODN3 antisense. (a) Western blot of 6A3-5/Osa2 protein expression, following 4 hours of PDGF-BB stimulation. Serum starved VSMC (0) were treated, first, by 6A3-5/Osa2-ODN3 sense (S), antisense (AS), mismatched (Mis), scrambled (Scr), or vehicle (NT) at $200 \mathrm{nM}$. (b) Northern blot of 6A3-5/Osa2 mRNA expression, following 4 hours of PDGF-BB stimulation. (c) Quantification of Northern blot results, which are representative of three independent experiments. (d) Western blot of 6A3-5/Osa2 protein expression, following 4 hours of PDGF-BB stimulation. Serum starved VSMC (0) were treated, first, by 6A3-5/Osa2-ODN3 sense or antisense at different concentrations $(25,100$, and $200 \mathrm{nM})$.

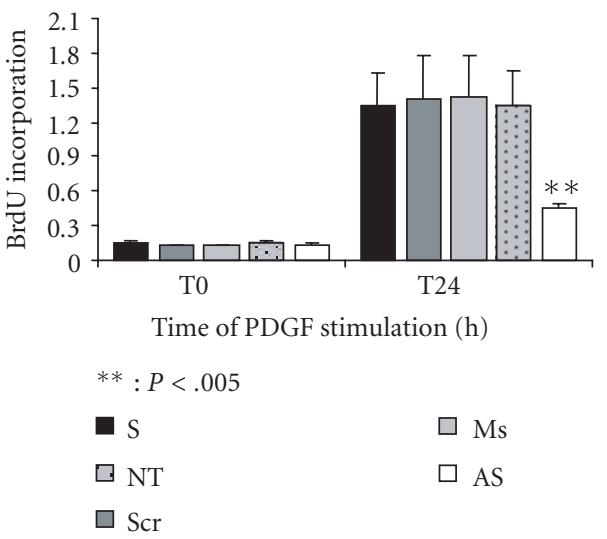

(a)

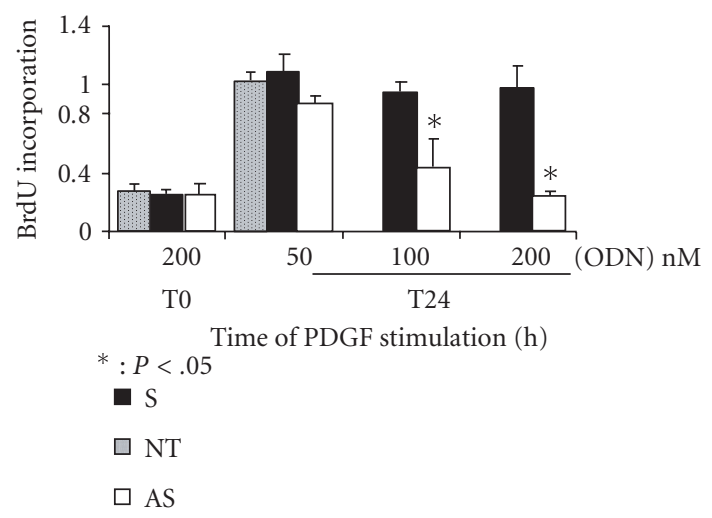

(b)

FIGURE 7: Inhibition of PDGF-stimulated VSMC proliferation by antisense ODN3. (a) Serum starved VSMC were treated by ODN3 sense, antisense, mismatched, and scrambled oligos at $200 \mathrm{nM}$ followed by PDGF-BB (20 ng/ml) stimulation for 0 or 24 hours in presence of BrdU. (b) Serum starved VSMC were treated by ODN3 sense and antisense (at 50, 100, or $200 \mathrm{nM}$ ) following 0 and 24 hours of PDGF-BB (20 ng/ml) stimulation. Untreated VSMC are used as controls of proliferation rate. The results are representative of four independent experiments. Data are presented as means \pm SEM.* $: P<.05$ versus nontransfected cells $(\mathrm{NT})$.

in calponin and SM22-alpha in both LH and LL animals (Figures 10(b), 10(c)). However, Vessel wall media thickness in LH and LL was not affected by such a treatment (Figure 11(c)).

\section{Expression of 6A3-5/Osa2 and vascular phenotype in Angiotensinll-perfused LH and LL rats}

Perindopril treatment, of the 2 strains, was followed by chronic perfusion of AngII which showed, over a period 


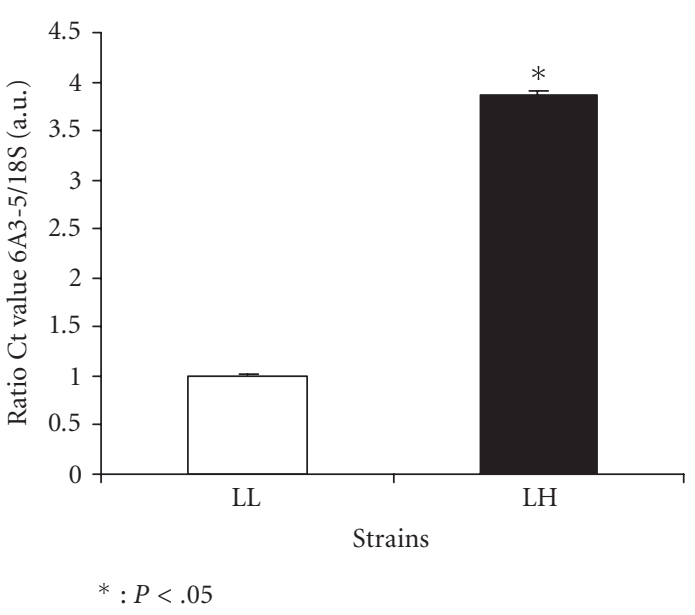

(a)

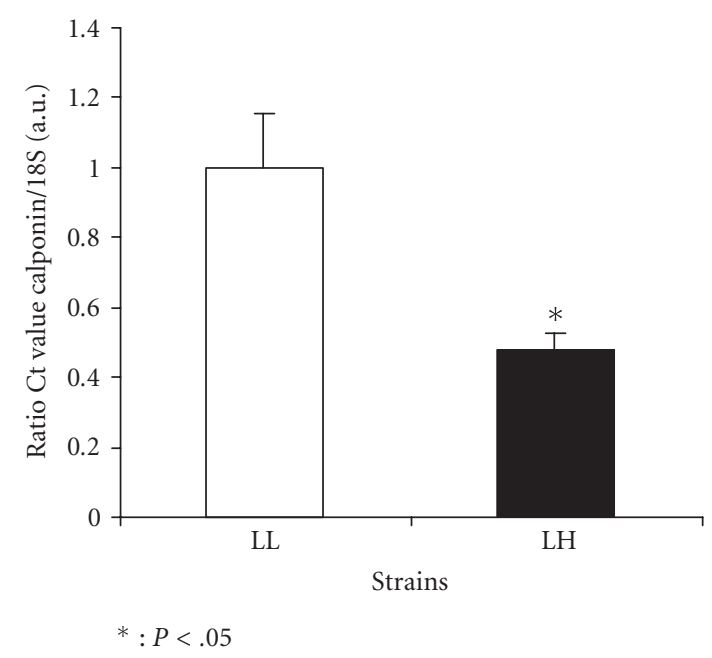

(b)

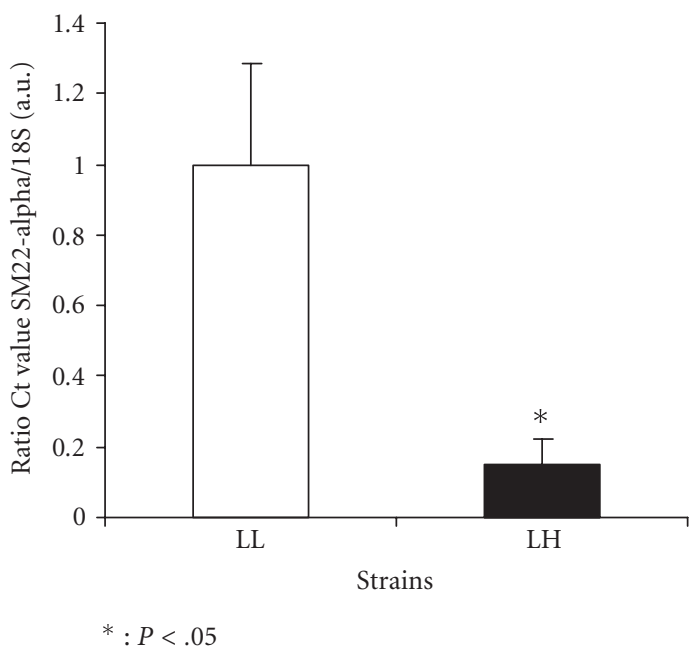

(c)

FIGURE 8: Expression of 6A3-5/Osa2 and vascular phenotype in LH versus LL rats. (a) 6A3-5/Osa2 aortic mRNA expression is significantly higher in hypertensive (LH) compared to normotensive (LL) rats. (b) Calponin (VSMC contractile phenotype marker) aortic mRNA gene expression is significantly reduced in LH versus LL rats. (c) SM22 alpha (VSMC contractile phenotype marker) aortic mRNA gene expression is significantly reduced in LH versus LL rats. Results are indicated as a ratio of mRNA expression in comparison to $18 \mathrm{~S}$ expression. Data are presented as means \pm SEM.* $: P<.05$ versus LL normotensive rats.

of 4 weeks, an increase of SBP in LH (from $134 \pm 1.84$ to $231 \pm 5.67 \mathrm{mmHg}$ ) and a steady SBP (from $104 \pm 2.39$ to $192 \pm 5.46 \mathrm{mmHg}$ ) in LL rats [16]. AngII induces a significant upregulation of aortic 6A3-5/Osa2 excised from hypertensive $(\mathrm{LH})$ rats in comparison to their unperfused controls (Figure 10(a)). Moreover, decrease in VSMC contractile markers, closely followed the hypertrophy state of the vessel wall in these two strains (Figures 10(b), 10(c)). In contrast, aortic 6A3-5/Osa2-gene expression was not modified in normotensive (LL) rats. One should note that AngII perfusion induced a significant aortic media hypertrophy in $\mathrm{LH}$ (Figure 11(c)) and to a much lesser extent in LL rats in comparison to their unperfused controls (Figure 11(c)).

\section{DISCUSSION}

This study reports the cloning and the characterization of a new gene (6A3-5/Osa2) overexpressed in proliferating rat vascular smooth muscle cells. Several lines of evidence show that this new gene is an early-gene activator that may be implicated in the control of VSMC activities.

6A3-5/Osa2 protein bears a DNA binding motif called ARID and two recently described conserved motifs, OHD1 and OHD2. These functional domains define the recently described Osa family of transcription modulator. Recently, Hurlstone et al [14] cloned the human homologue of 6A35 and showed that the OHD2 motif is necessary for binding 
LH

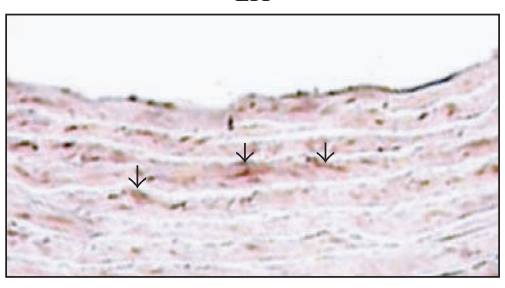

(a)

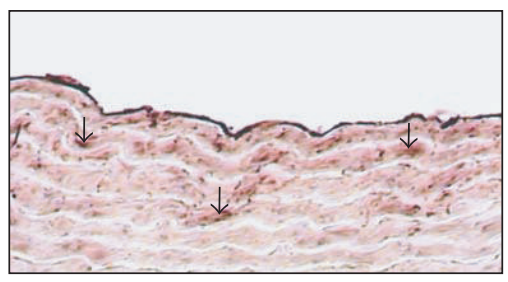

(c)
LL

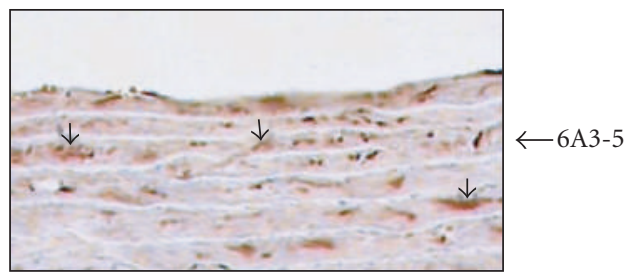

(b)

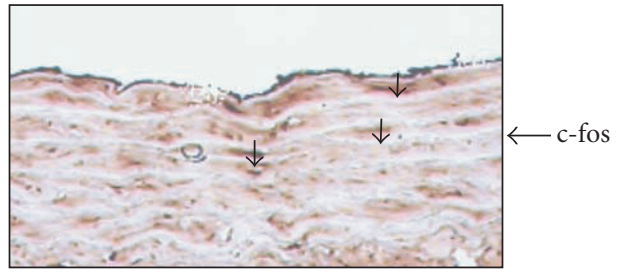

(d)

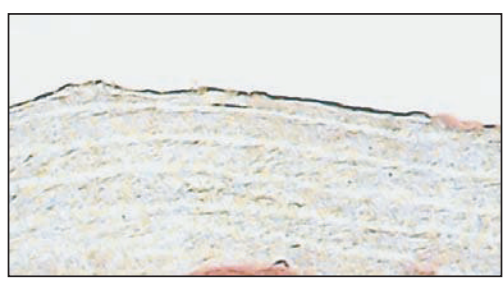

(e)

FIGURE 9: Localization of 6A3-5/Osa2 in aortas from LH and LL rats. (a) 6A3-5/Osa2 labelling is observed in SMC of the inner media from AngII-perfused LH rats (X40). (b) 6A3-5/Osa2 labelling is observed in SMC of the inner media from AngII-perfused LL rats. (c) c-fos antibody showed a similar localization to 6A3-5/Osa2 in LH rats. (d) c-fos antibody showed a similar localization to 6A3-5/Osa2 in LL rats. (e) Negative control showed no labelling. Similar localization and labelling was observed for 6A3-5/Osa2 for all tested aortas (data not shown).

BRG-1 (Brahma-related gene-1), a key catalytic component of the SWI/SNF-A chromatin remodelling complex. In contrast to other ARID proteins, Osa proteins show no sequence preference for AT rich sites. Nonetheless, work using Drosophila suggest that Osa proteins may participate in targeting SWI/SNF to a subset of promoters in vivo and induce the activation or repression of target gene expression. Prior to our study, no Osa protein had been described in vascular cells, and very little is known about the function of these proteins in mammals.

In this study, we have observed, in a similar way to c-fos, an early upregulation of 6A3-5/Osa2 soon after mitogenic stimulation of human or rat VSMC by PDGF-BB. Increased activity of the PDGF signalling pathway has been implicated as a contributing factor in the progression of atherosclerosis or restenosis. PDGF induces activation and phosphorylation of several cytosolic signalling molecules and nuclear transcription factors, including Egr-1 (early growth response-1), Ets-1, $c$-fos, and $c$-jun, which stimulate expression of their target genes. These data indicate that $6 \mathrm{~A} 3-5 / \mathrm{Osa} 2$ is an early PDGF-responding gene potentially implicated in VSMC proliferation. To validate this hypothesis, we generated four spe- cific sets of ODN antisense directed against 6A3-5/Osa2. Only one of these, ODN3, is able to inhibit 6A3-5/Osa2 expression at the mRNA and the protein level in dose and sequence-dependant manner. It is interesting to note that ODN3 targets the ATG initiation site. Previous studies have demonstrated that such targeting is very effective in inhibiting gene expression by antisense phosphorothioate oligonucleotides. Indeed, ODN controls used in the present study indicated that 6A3-5/Osa2 RNA and protein depletion was due to a sequence-specific antisense effect, as neither the sense nor the scrambled or mismatched control ODNs caused 6A3-5/Osa2 depletion. Moreover, we observed no effect on p53 gene expression following Osa2 inhibition, suggesting that $\mathrm{ODN}_{\mathrm{AS}}$ inhibit selectively 6A3-5 expression. We then used ODN3 in association with BrdU incorporation assays to assess the role of 6A3-5/Osa2 in VSMC proliferation. ODN3 antisense was able to significantly reduce proliferation of PDGF-stimulated VSMC in a dose and sequence-dependent manner. Recently, Watanabe et al [22] produced the first evidence that an ARID protein family member is implicated in differentiation and control of VSMC proliferation. Their study showed that overexpression of Mrf2 induces expression 


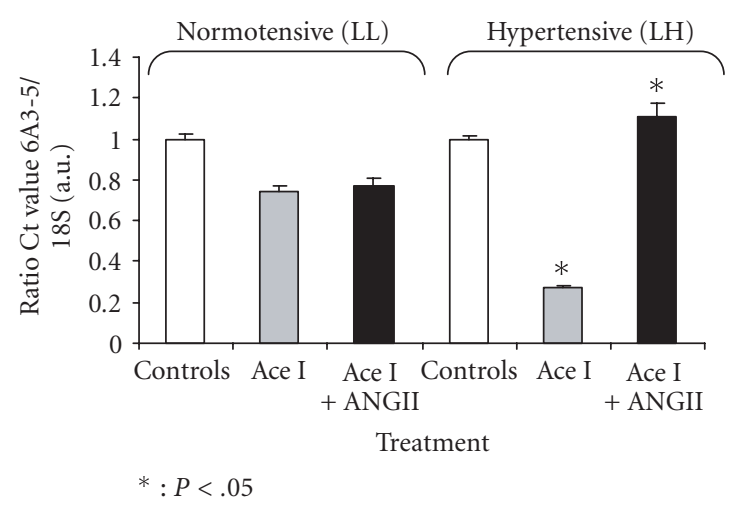

(a)

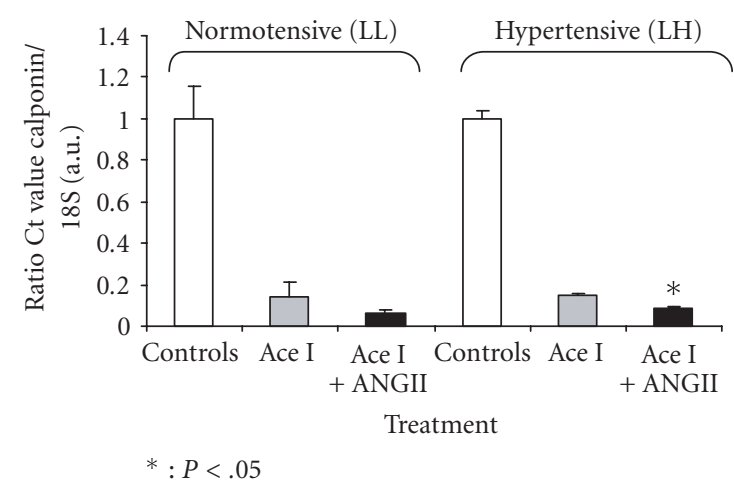

(b)

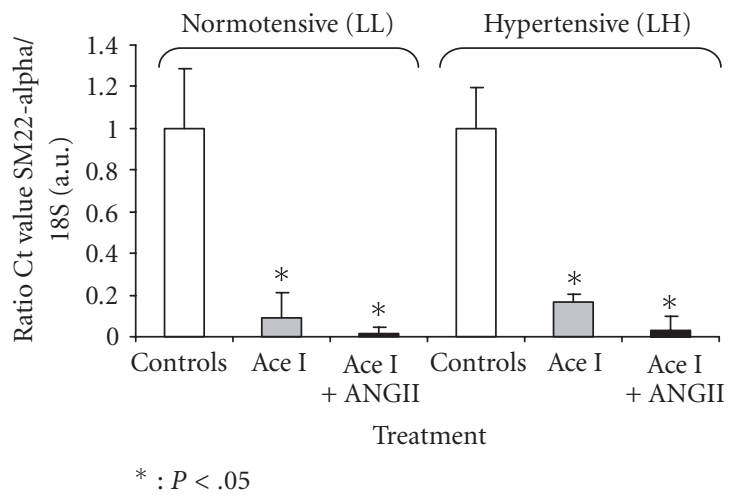

(c)

FIGURE 10: Expression of 6A3-5/Osa2 and vascular phenotype in ACE inhibitor treated LH and LL rats. (a) 6A3-5/Osa2 aortic mRNA expression in untreated LH and LL rats (controls) was compared to ACE inhibitor treated rats in the absence (Ace I) or presence of perfused ANGII (Ace I + ANGII). 6A3-5/Osa2 gene expression is downregulated by the ACE inhibitor and upregulated by exogenous AngiotensinII in LH rats. (b) Calponin gene expressions were quantified in these same animals. (c) SM22 alpha gene expressions were also quantified in these same animals. Results are indicated as a ratio of 6A3-5/Osa2 mRNA expression in comparison to $18 \mathrm{~S}$ expression. Data are presented as means \pm SEM. $^{*}: P<.05$ versus controls untreated rats, for each strain.

of specific smooth muscle marker, such as alpha-actin and SM-22alpha. Interestingly, in contrast to 6A3-5/Osa2, Mrf2 retarded cellular proliferation. It is interesting to note that Mrf-2 binds a specific DNA sequence (AATA(C/T)) in contrast to Osa proteins. The apparent functional divergence in regard to cellular proliferation between the two ARIDbearing proteins could be linked to different properties of their DNA binding activities. The mechanism by which 6A3$5 / \mathrm{Osa} 2$ influences cell proliferation is unknown. However, human Osa2 was recently shown, to stimulate transcription as a cofactor of glucocorticoid receptor-dependent transcriptional activation in cultured mammalian cells [25]. Interestingly, glucocorticoids are known to modulate proliferation and expression of some target genes in VSMC (such as $\mathrm{I} \kappa \mathrm{B}, \mathrm{NaKATPase}$, adrenomedullin). Further investigation will be necessary to investigate by which molecular mechanisms, that is, by which target genes 6A3-5/Osa2 influences VSMC proliferation.

In a similar way to PDGF, we have previously observed an early upregulation of 6A3-5/Osa2 in cultured rat VSMC in response to AngII [15]. Several signalling responses are shared between PDGF and AngII activation. Indeed, AngII stimulation of VSMC is associated, in a similar manner to PDGF, with an upregulation of early activated genes such as c-fos and c-myc and growth factors such as PDGF and bFGF [9]. ACE inhibition by perindopril induces a reduction of cfos and c-jun expression in response to balloon injury [26]. In vitro study has shown a link between AngII receptor and PDGF $\beta$ receptor in cultured VSMC [27]. Moreover, AngII has recently been reported to transactivate the PDGF $\beta$ receptor by cross-talk in stroke-prone SHR rats by comparison, Wistar-Kyoto rats their normotensive controls, did not show this effect [28]. In this study, hypertensive rats (LH) had significantly higher aortic 6A3-5/Osa2 gene expression levels in comparison to normotensive rats (LL). Moreover, while perindopril treatment reduced blood pressure in these 2 strains, it only affected 6A3-5/Osa2-gene expression in LH but not LL. Finally, exogenous AngII perfusion in the presence of ACE inhibitor increased blood pressure levels in both strains but increased 6A3-5/Osa2-gene expression only in LH 


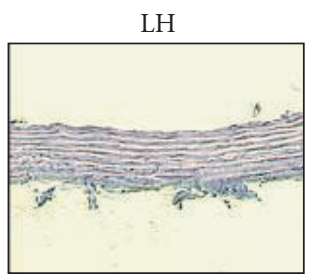

(a)

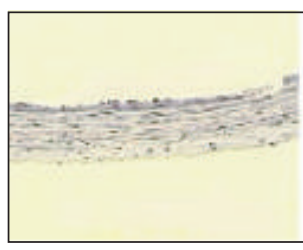

(b)

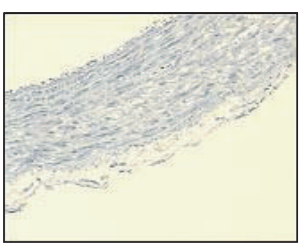

(c)

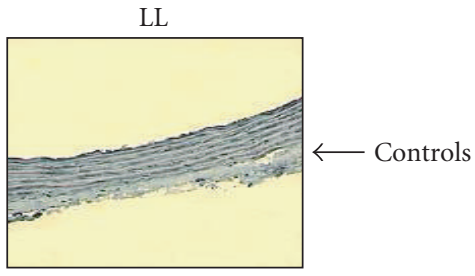

(d)

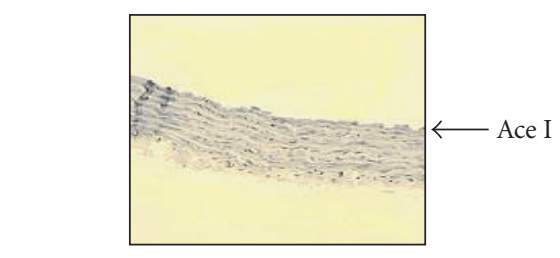

(e)

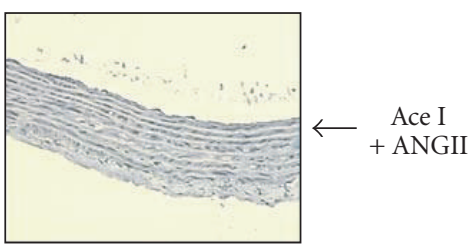

(f)

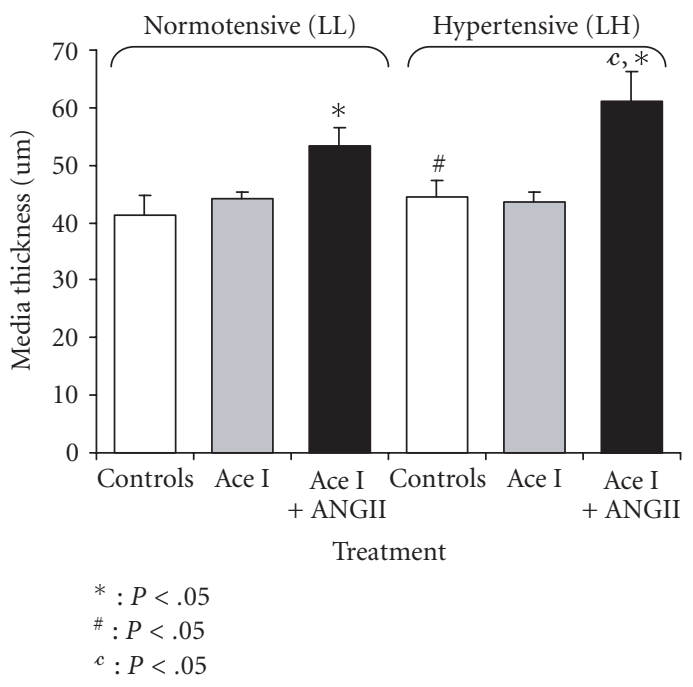

(g)

FIGURe 11: Analysis of media hypertrophy. Media thickness was determined, following haematoxylin/eosin staining of aorta sections. (a) Control LH rats were studied for their media thickness. (b) AceI treated LH rats were also analysed. (c) AceI and ANGII treated LH rats. (d) Control LL rats. (e) AceI treated LL rats. (f) AceI + ANGII treated LL rats. (g) Quantification of the above data is presented as means \pm SEM $^{*}: P<.05$ versus controls for each strain. ${ }^{\#}: P<0.05$ versus normotensive controls rats, ${ }^{c}: P<.05$ versus AngII-perfused normotensive rats.

but not LL. Interestingly, Kim et al [28] have reported that treatment of SHR rats with perindopril significantly reduced aortic PDGF- $\beta$ receptor phosphorylation and ERKinase activity which is restored by chronic (but not acute) infusion of AngII. It is known that PDGF $\beta$ receptor is chronically activated in SHR compared to Wistar-Kyoto rats.

While LH rats present a higher blood pressure than LL rats, similar levels of plasma AngII were reported [29]. Interestingly, results by Lantelme et al [30], have shown that inhibition of the renin-angiotensinII system in newborn $\mathrm{LH}$ rats prevents the development of hypertension. It is conceivable that VSMC of LH rats are very much more sensitive to
AngII compared to LL. Such hypersensitivity has been reported for VSMC, isolated from SHR rats, which show abnormal growth in vitro with accelerated entry into $S$ phase of cell cycle and increased cdk2 activity in comparison to VSMC from Wistar-Kyoto rats [31]. Aortic gene expression of $6 \mathrm{~A} 3-5 / \mathrm{Osa} 2$ is significantly increased in $\mathrm{LH}$ compared to LL rats. Such enhanced expression of 6A3-5/Osa2 gene in $\mathrm{LH}$ rats may be linked to the potential hypersensitivity of the VSMC that not only results in increased blood pressure but modified phenotype gene markers and media hypertrophy. On treatment with an ACE inhibitor, LH rats show a significant reduction in aortic 6A3-5/Osa2 expression, not 
observed in LL rats, that is presumably due to the hypersensitivity of the VSMC to AngII. Chronic perfusion of AngII, in the presence of an ACE inhibitor, induces a significant increase in 6A3-5/Osa2 expression in LH but not in LL rats. Sabri et al [32], have shown that AngII perfusion induces a reversion of VSMC to an immature phenotype. Similarily to AngII, PDGF under in vitro conditions induces suppression of smooth muscle-specific gene ( $\square$-actin and SM22alpha) through activation of Pi3K/Akt signalling pathways and subcellular redistribution of serum response factor [33]. One should also note that higher glucocorticoid plasma levels are observed in LH strains in response to AngII [16]. As previously indicated, 6A3-5/Osa2 has been implicated as a cofactor of glucocorticoid receptor-dependent transcription [25]. The overall data in this study strongly suggests that the potential hypersensitivity of VSMC, in LH rats, not only controls blood pressure levels but also 6A3-5/Osa2 expression, gene markers of VSMC phenotype, and media hypertrophy.

\section{ACKNOWLEDGMENTS}

This work was supported by grants from INSERM and the Mary and Gary Weston foundation and by a PhD fellowship from the French Ministry of Research for GG. We would like also to thank Professor JB Michel and Dr MC Bourdillon for kindly providing us with human and rat vascular smooth muscle cells, respectively, as well as Dr Olga Mattewa for her help in generation of antisense oligonucleotides.

\section{REFERENCES}

[1] Ross R. The pathogenesis of atherosclerosis: a perspective for the 1990s. Nature. 1993;362(6423):801-809.

[2] Schwartz SM, Campbell GR, Campbell JH. Replication of smooth muscle cells in vascular disease. Circulation Research. 1986;58(4):427-444.

[3] Hughes AD, Clunn GF, Refson J, Demoliou-Mason C. Platelet-derived growth factor (PDGF): actions and mechanisms in vascular smooth muscle. General Pharmacology. 1996;27(7):1079-1089.

[4] Daemen MJ, Lombardi DM, Bosman FT, Schwartz SM. Angiotensin II induces smooth muscle cell proliferation in the normal and injured rat arterial wall. Circulation Research. 1991;68(2):450-456.

[5] Touyz RM. The role of angiotensin II in regulating vascular structural and functional changes in hypertension. Current Hypertension Reports. 2003;5(2):155-164.

[6] Sharma RV, Bhalla RC. PDGF-induced mitogenic signaling is not mediated through protein kinase $\mathrm{C}$ and $\mathrm{c}$-fos pathway in VSM cells. American Journal of Physiology. 1993;264(1 pt 1):C71-C79.

[7] Hultgardh-Nilsson A, Cercek B, Wang J-W, et al. Regulated expression of the Ets-1 transcription factor in vascular smooth muscle cells in vivo and in vitro. Circulation Research. 1996;78(4):589-595.

[8] Brand K, Page S, Rogler G, et al. Activated transcription factor nuclear factor-kappa B is present in the atherosclerotic lesion. Journal of Clinical Investigation. 1996;97(7):1715-1722.

[9] Griendling KK, Alexander RW. Cellular mechanisms of angiotensin II action. In: Swales JD, ed. Textbook of Hypertension. Oxford, UK: Blackwell; 1994:244-253.
[10] Zibara K, Garin G, McGregor JL. Identification, structural, and functional characterization of a new early gene (6A3$5,7 \mathrm{~kb}$ ): implication in the proliferation and differentiation of smooth muscle cells. Journal of Biomedicine and Biotechnology. 2005;2005(3):254-270.

[11] Wilsker D, Patsialou A, Dallas PB, Moran E. ARID proteins: a diverse family of DNA binding proteins implicated in the control of cell growth, differentiation, and development. Cell Growth and Differentiation. 2002;13(3):95-106.

[12] Gregory SL, Kortschak RD, Kalionis B, Saint R. Characterization of the dead ringer gene identifies a novel, highly conserved family of sequence-specific DNA-binding proteins. Molecular and Cellular Biology. 1996;16(3):792-799.

[13] Campbell JH, Campbell GR. Phenotypic modulation of smooth muscle cells in primary culture. In: Campbell JH, Campbell GR, eds. Vascular Smooth Muscle in Culture. 1st ed. Boca Raton, Fla: CRC Press; 1987.

[14] Hurlstone A, Olave IA, Barker N, Van Noort M, Clevers H. Cloning and characterization of hELD/0SA1, a novel BRG1 interacting protein. Biochemical Journal. 2002;364(1):255-264.

[15] Garin G, Badid C, McGregor B, et al. Ischemia induces early expression of a new transcription factor (6A3-5) kidney vascular smooth muscle cells: studies in rat and human renal pathology. American Journal of Pathology. 2003;163(6):2485-2494.

[16] Aguilar F, Lo M, Claustrat B, Saez JM, Sassard J, Li JY. Hypersensitivity of the adrenal cortex to trophic and secretory effects of angiotensin II in Lyon genetically-hypertensive rats. Hypertension. 2004;43(1):87-93.

[17] Kozmik Z, Machoň O, Králová J, Krešlová J, Pačes J, Vlček C. Characterization of mammalian orthologues of the Drosophila osa gene: cDNA cloning, expression, chromosomal localization, and direct physical interaction with brahma chromatinremodeling complex. Genomics. 2001;73(2):140-148.

[18] Treisman JE, Luk A, Rubin GM, Heberlein U. Eyelid antagonizes wingless signaling during Drosophila development and has homology to the bright family of DNA-binding proteins. Genes and Development. 1997;11(15):1949-1962.

[19] Peterson CL, Herskowitz I. Characterization of the yeast SWI1, SWI2, and SWI3 genes, which encode a global activator of transcription. Cell. 1992;68(3):573-583.

[20] Gregory SL, Kortschak RD, Kalionis B, Saint R. Characterization of the dead ringer gene identifies a novel, highly conserved family of sequence-specific DNA-binding proteins. Molecular and Cellular Biology. 1996;16(3):792-799.

[21] Webb CF. The transcription factor, Bright and immunoglobulin heavy chain expression. Immunologic Research. 2001;24(2):149-161.

[22] Watanabe M, Layne MD, Hsieh C-M, et al. Regulation of smooth muscle cell differentiation by AT-rich interaction domain transcription factors Mrf2 $\alpha$ and Mrf2 $\beta$. Circulation Research. 2002;91(5):382-389.

[23] Shanahan CM, Weissberg PL. Smooth muscle cell heterogeneity: patterns of gene expression in vascular smooth muscle cells in vitro and in vivo. Arteriosclerosis, Thrombosis, and Vascular Biology. 1998;18(3):333-338.

[24] Blaes N, Bourdillon MC, Daniel-Lamaziere JM, Michaille JJ, Andujar M, Covacho C. Isolation of two morphologically distinct cell lines from rat arterial smooth muscle expressing high tumorigenic potentials. In Vitro Cellular \& Developmental Biology. 1991;27(9):725-734.

[25] Inoue H, Furukawa T, Giannakopoulos S, Zhou S, King DS, Tanese N. Largest subunits of the human SWI/SNF 
chromatin-remodeling complex promote transcriptional activation by steroid hormone receptors. Journal of Biological Chemistry. 2002;277(44):41674-41685.

[26] Van Belle E, Bauters C, Wernert N, et al. Angiotensin converting enzyme inhibition prevents proto-oncogene expression in the vascular wall after injury. Journal of Hypertension. 1995;13(1):105-112.

[27] Linseman DA, Benjamin CW, Jones DA. Convergence of angiotensin II and platelet-derived growth factor receptor signaling cascades in vascular smooth muscle cells. Journal of Biological Chemistry. 1995;270(21):12563-12568.

[28] Kim S, Zhan Y, Izumi Y, Yasumoto H, Yano M, Iwao H. In vivo activation of rat aortic platelet-derived growth factor and epidermal growth factor receptors by angiotensin II and hypertension. Arteriosclerosis, Thrombosis, and Vascular Biology. 2000;20(12):2539-2545.

[29] Vincent M, Sassard J. Lyon strains. In: Swales JD, ed. Textbook of Hypertension. Oxford, UK: Blackwell; 1994:455-456.

[30] Lantelme P, Lo M, Luttenauer L, Sassard J. Pivotal role of the renin-angiotensin system in Lyon hypertensive rats. American Journal of Physiology. 1997;273(5 pt 2):R1793-R1799.

[31] Tanner FC, Greutert H, Barandier C, Frischknecht K, Lüscher TF. Different cell cycle regulation of vascular smooth muscle in genetic hypertension. Hypertension. 2003;42(2):184-188.

[32] Sabri A, Levy BI, Poitevin P, et al. Differential roles of $\mathrm{AT}_{1}$ and $\mathrm{AT}_{2}$ receptor subtypes in vascular trophic and phenotypic changes in response to stimulation with angiotensin II. Arteriosclerosis, Thrombosis, and Vascular Biology. 1997;17(2):257264.

[33] Kaplan-Albuquerque N, Garat C, Desseva C, Jones PL, Nemenoff RA. Platelet-derived growth factor-BB-mediated activation of Akt suppresses smooth muscle-specific gene expression through inhibition of mitogen-activated protein kinase and redistribution of serum response factor. Journal of Biological Chemistry. 2003;278(41):39830-39838. 

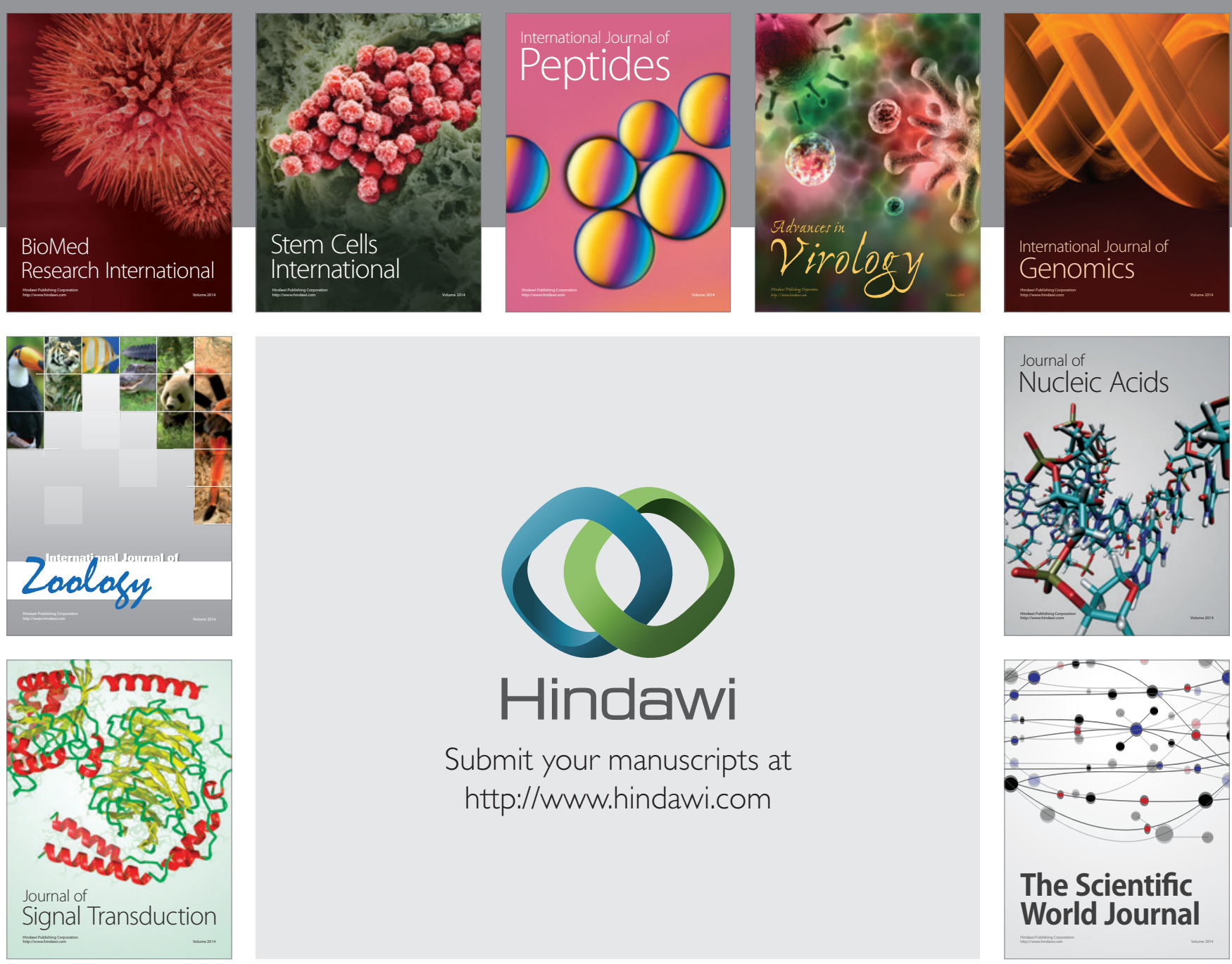

Submit your manuscripts at

http://www.hindawi.com
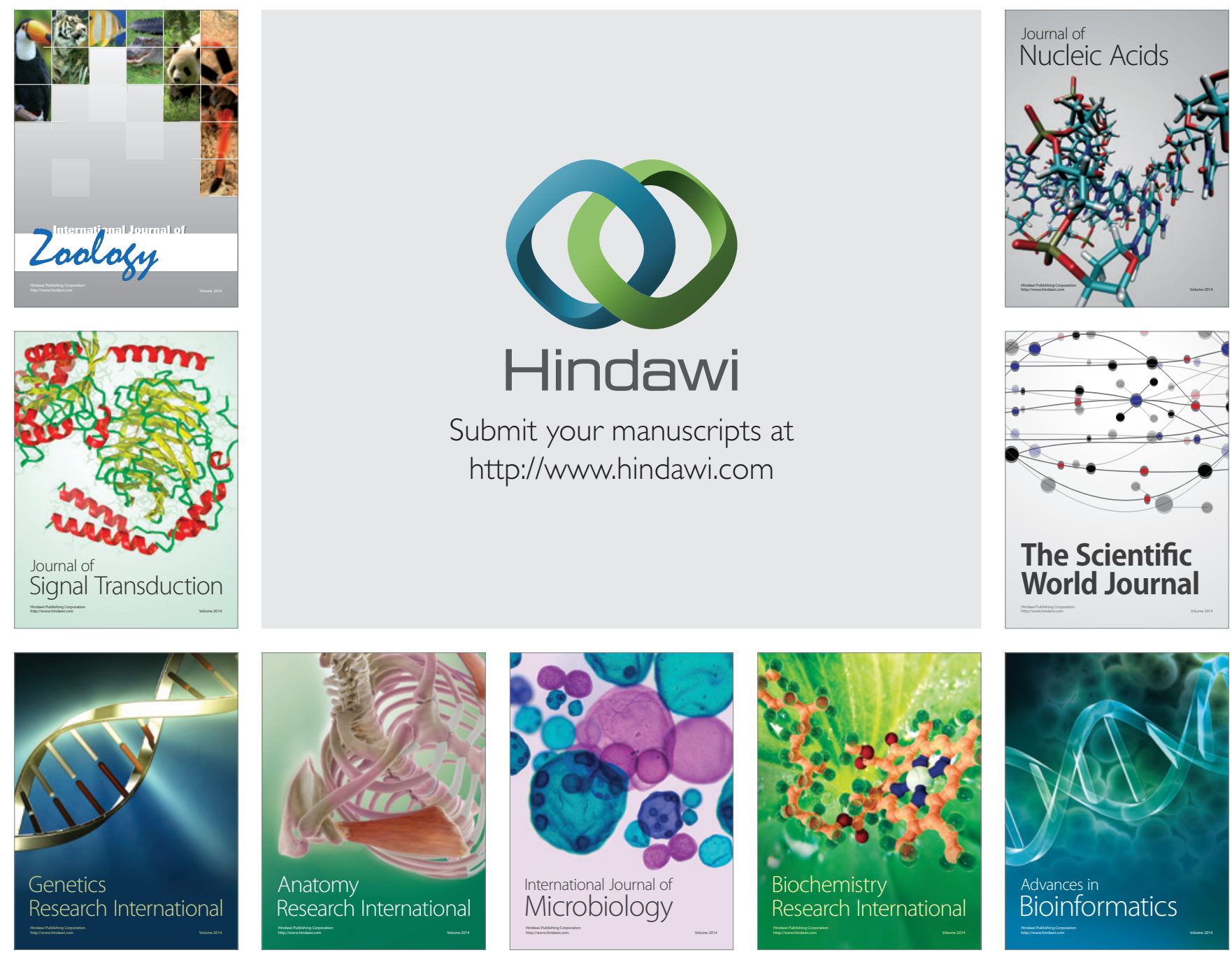

The Scientific World Journal
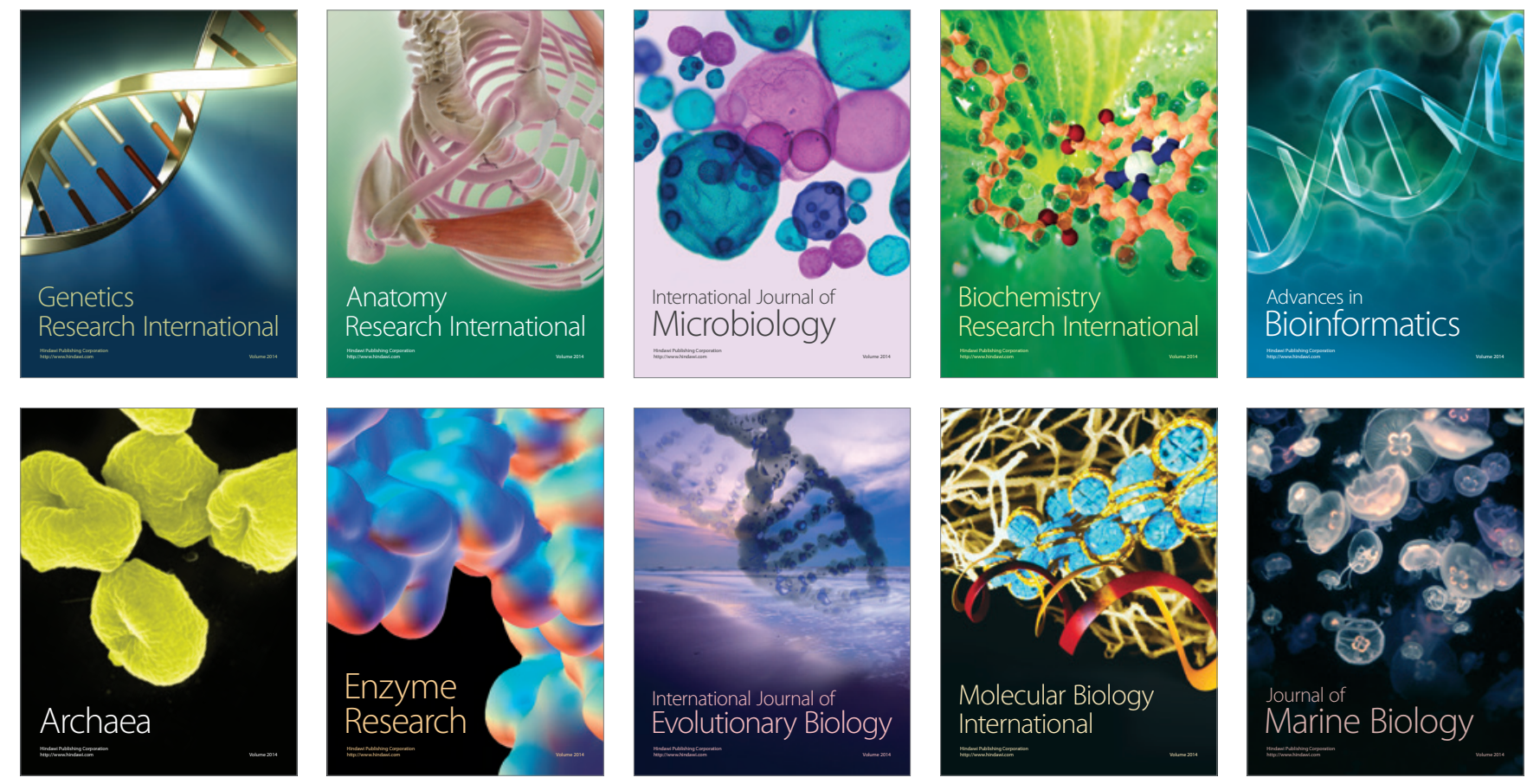